\title{
Ordered Median Hub Location Problems with Capacity Constraints
}

\author{
J. Puerto $^{a} \quad$ A.B. Ramos $^{a} \quad$ A.M. Rodríguez-Chía ${ }^{b} \quad$ M.C. Sánchez-Gil ${ }^{b}$ \\ ${ }^{a}$ Facultad de Matemíticas. Universidad de Sevilla, Spain. \\ ${ }^{b}$ Facultad de Ciencias. Universidad de Cádiz, Spain.
}

March 17, 2015

\begin{abstract}
The Single Allocation Ordered Median Hub Location problem is a recent hub model introduced in [36] that provides a unifying analysis of a wide class of hub location models. In this paper, we deal with the capacitated version of this problem, presenting two formulations as well as some preprocessing phases for fixing variables. In addition, a strengthening of one of these formulations is also studied through the use of some families of valid inequalities. A battery of test problems with data taken from the AP library are solved where it is shown that the running times have been significantly reduced with the improvements presented in the paper.
\end{abstract}

\section{Introduction}

Network design problems are among the most interesting models in combinatorial optimization. In the last years researchers have devoted a lot of attention to a particular member within this family, namely the hub location problem, that combines network design and location aspects of supply chain models, see the surveys [1, 7, 9]. The main advantage of using hubs in distribution problems is that they allow to consolidate shipments in order to reduce transportation costs by applying economies of scale; which are naturally incorporated to the models through discount factors. Hub location problems have been studied from different per- 
spectives giving rise to a number of papers considering different criteria to be optimized: the minimization of the overall transportation cost (sum) (see [10, 6, 20, 27, 29, 30, 31]), the minimization of the largest transportation cost or the coverage cost $(4,4,24,25,26,34,40,41)$, et cetera.

Apart from the choice of the optimization criterion, another crucial aspect in the literature on hub location, and in general on any location problem, is the assumption of capacity constraints. One can recognize that although this assumption implies more realistic models, the difficulty to solve them also increases in orders of magnitude with respect to their uncapacitated counterpart. In many cases new formulations are needed and a more specialized analysis is often required to solve even smaller sizes than those previously addressed for the uncapacitated versions of the problems. For this reason, capacitated versions of hub location problems have attracted the interest of locators in the last years, see [2, 5, 11, 13, 14, 15, 16, 20, 29]. In the same line, we also mention some other references related with congestion at hubs, as congestion acts as a limit on capacity, see [17, 18, 28].

An interesting version of hub location model is the Capacitated Hub Location Problem with Single Allocation (CSA-HLP), see [11, 13, 20]. In this context, single allocation means that incoming and outgoing flow of each site must be shipped via the same hub. In contrast to single allocation models, where binary variables are required in the allocation phase, multiple allocation allows different delivery patterns which in turns implies the use of continuous variables simplifying the problems. The CSA-HLP model incorporates capacity constraints on the incoming flow at the hubs coming from origin sites or even simpler, on the number of non-hub nodes assigned to each hub. The inclusion of capacity constraints make these models challenging from a theoretical point of view. Regarding its applicability we cite one example described in Ernst at al. [20] based on a postal delivery application, where a set of $n$ postal districts (corresponding to postcode districts represented by nodes) exchange daily mail. The mail between all the pairs of nodes must be routed via one or at most two mail consolidation centers (hubs). In order to meet time constraints, only a limited amount of mail could be sorted at each sorting center (mail is just sorted once, when it arrives to the first hub from origin sites). Hence, there are capacity restrictions on the incoming mail that must be sorted. 
The problem requires to choose the number and location of hubs, as well as to determine the distribution pattern of the mail.

The CSA-HLP has received less attention in the literature than its uncapacitated counterpart. Campbell [5] presented the first integer Mathematical Programming formulation for the Capacitated Hub Location Problem. This formulation was strenghthened by Skorin-Kapov et al. [39]. Ernst and Krishnamoorthy [20], proposed a new model involving three-index continuous variables and developed a solution approach based on Simulated Annealing where the bounds obtained are embedded in a branch-and-bound procedure devised for solving the problem optimally. Recently, Correia et al. [13] have shown that this formulation may be incomplete and an additional set of inequalities is proposed to assure the validity of the model in all situations. A new formulation using only two indices variables was proposed by Labbé et al. [27, where a polyhedral analysis and new valid inequalities were addressed. Although this formulation has only a quadratic number of variables, it has an exponential number of constraints, and to solve it the authors developed a branch-and-cut algorithm based on their polyhedral analysis. Contreras et al. [11] presented for the same problem a Lagrangean relaxation enhanced with reduction tests that allows the computation of tight upper and lower bounds for a large set of instances.

In two recent papers, [36, 37, a new model of hub location, namely the Single Allocation Ordered Median hub location problem (SA-OMHLP), has been introduced and analyzed. This problem can be seen as a powerful tool from a modeling point of view since it allows a common framework to represent many of the previously considered criteria in the literature of hub location. Moreover, this approach is a natural way to represent the differentiation of the roles played by the different parties (origins, hubs and destinations) in logistics networks [21, 22, 23, 32]. This model does not assume, in advance, any particular structure on the network $([11,12])$. Instead of that this structure is derived from the choice of the parameters defining the objective function. Apart from the above mentioned characteristics, ordered median objectives are also useful to obtain robust solutions in hub problems by applying $k$ centrum, trimmed-mean or anti-trimmed-mean criteria. It is worth mentioning that although it is called single allocation, its meaning slightly differs from the classical interpretation in 
hub location where each site is allocated to just one hub and all the incoming and outgoing flow to-from this site is shipped via the same link (the one joining this site and its allocated hub). In this model, single allocation means that all the outgoing flow is delivered through the same hub, but the incoming flow can come from different hubs. Actually, this is a mixed model and basically the same situation described above, about postal deliveries, naturally fits in this framework assuming that letters from the same origin should be sorted, with respect to their destinations, in the same place and from there they are delivered via their cheapest routes. Observe that in this scheme it is also natural that incoming flow in a final destination comes from different hubs.

The SA-OMHLP distinguishes among segmented origin-destination deliveries giving different scaling factors to the origin-hub, hub-hub and hub-destination links. The cost of each origin-first hub link is scaled by a factor that depends on the position of this cost in the ordered sequence of costs from each origin to its corresponding first hub [3, 32, 35]. Moreover, the overall interhub cost and hub-destination cost are multiplied by other economy of scale factors. The goal is to minimize the overall shipping cost under the above weighting scheme. The reader may note that the first type of scaling factors mentioned above adds a "sorting" problem to the underlying hub location model, making its formulation and solution much more challenging. This model and two different formulations were introduced in [35] while a specialized B\&B\&Cut algorithm was developed in [36, 38]. None of those formulations could handle capacities since the computation burden of the problems were highly demanding. Thus, the SA-OMHLP with capacity constraints, i.e. Capacitated Single Allocation Ordered Median hub location problem (CSA-OMHLP) is currently an open line of research for further analysis.

In this paper, we analyze in depth the CSA-OMHLP trying to obtain a better knowledge and alternative ways to solve it. Thus, the contributions of this paper are threefold. First, it combines for the first time three challenging elements in location analysis: hub facilities, capacities and ordered median objectives; proposing a promising IP formulation which remarkably reduces the number of decision variables. Second, this paper strengthens that formulation with variable fixing and some families of valid inequalities that have not been 
considered before. Finally, despite the difficulty of considering simultaneously capacitated models, hubs and ordering, the techniques proposed in this paper allow to solve instances of similar sizes to those already considered in the literature for simpler models (uncapacitated and multiple allocation [22]).

The paper is organized as follows: in Section 2 we will provide, first, a MIP formulation for the capacitated version of the problem extending the one in [36] and then another formulation in the spirit of [37] where the number of variables has been considerably reduced with respect to the previous one. Section 3 strengthens the latter formulations with variable fixing and several new families of valid inequalities. In Section 4, the effectiveness of the proposed methodology is shown with an extensive computational experience comparing the performance of the two formulations and the strengthening proposed along the paper. Finally, the paper ends with some conclusions.

\section{Model and MIP formulations}

The goal of this paper is to analyze the CSA-OMHLP. For this reason, we elaborate from the most promising formulations of the non-capacitated version of that problem, namely the so called radius (covering) formulations, see [36, 37]. In order to be self-contained and for the sake of readability, we include next a concise description of these formulations in their application to the capacitated problem.

Let $A=\{1, \ldots, n\}$ be a set of $n$ client sites, where each site is collecting or gathering some commodity that must be sent to the remaining ones. It is assumed, without loss of generality, that the set of candidate sites for establishing hubs is also A. Let $w_{j m} \geq 0$ be the amount of commodity to be supplied from the $j$-th to the $m$-th site for all $j, m \in A$, and let $W_{j}=\sum_{m \in A} w_{j m}$ be the total amount of commodity to be sent from the $j$-th site. Let $c_{j m} \geq 0$ denote the unit cost of sending commodity from site $j$ to site $m$ (not necessarily satisfying the triangular inequality). It is assumed free self-service, i.e., $c_{j j}=0, \forall j \in A$.

Let $p \leq n$ be the number of hubs to be located and let $b_{j}$ be the capacity of a hub located at site $j$, with $j \in A$. A solution for the problem is a set of sites $X \subseteq A$ with $|X|=p$ and enough capacity to cover the flow coming from the sites; plus a set of links connecting pairs 
(flow patterns) of sites $j, m$ for all $j, m \in A$. Moreover, it is assumed that the flow pattern between each pair of sites traverses at least one and no more than two hubs from $X$.

As it was mentioned in Section 1, the main advantage of using hubs is to reduce costs by applying economies of scale to consolidated flows in some part of the network. In this model the transportation cost is decoupled into the three differentiated possible links: origin site-first hub, hub-to-hub, and hubs-final destination. These transportation costs are scaled in a different way. The model weights origin site-first hub transportation costs by using parameters $\lambda=\left(\lambda_{1}, \ldots, \lambda_{n}\right)$, with $\lambda_{i} \geq 0 \forall i \in A$, depending on their ordered rank values. This is, let $\hat{c}_{j k}$ be the cost of the overall flow sent from the origin site $j$ if it were delivered via the first hub $k$, i.e. $\hat{c}_{j k}:=c_{j k} W_{j}, j, k \in A$. Next, if $\hat{c}_{j k}$ were ranked in the $i$-th position among all these costs, then this term would be scaled by $\lambda_{i}$ in the objective function. For the two remaining links there is a compensation factor $0<\mu<1$ for the deliveries between hubs, and another one $0<\delta<1, \mu<\delta$, for the deliveries between hubs and final destination sites. These parameters may imply that, even in the case where the costs satisfy the triangle inequality, using a second hub results in a cheaper connection than going directly from the first hub to the final destination. Actually, it represents the application of the economy of scale by the consolidation of flow in the hubs.

In the following we present a first valid formulation of the CSA-OMHLP, based on covering variables (the reader is referred to [36, 37] for further details). Sorting the different delivery costs values $\left(\hat{c}_{j k}\right)$ for $j, k \in A$, in increasing order, we get the ordered cost sequence:

$$
\hat{c}_{(1)}:=0<\hat{c}_{(2)}<\cdots<\hat{c}_{(G)}:=\max _{1 \leq j, k \leq n}\left\{\hat{c}_{j k}\right\}
$$

where $G$ is the number of different elements of the above cost sequence. For convenience we consider $\hat{c}_{(0)}:=0$.

For $i \in A$ and $h=1, \ldots, G$, we define the following set of covering variables,

$$
\bar{u}_{i h}:= \begin{cases}1, & \text { if the } i \text {-th smallest allocation cost is at least } \hat{c}_{(h)}, \\ 0, & \text { otherwise. }\end{cases}
$$

Clearly, the $i$-th smallest allocation cost is equal to $\hat{c}_{(h)}$ if and only if $\bar{u}_{i h}=1$ and $\bar{u}_{i, h+1}=0$. 
In addition, this formulation uses two more sets of variables:

$$
x_{j k}= \begin{cases}1, & \text { if the commodity sent from origin site } j \text { goes first to the hub } k, \\ 0, & \text { otherwise. }\end{cases}
$$

$s_{k \ell m}=$ flow that goes through a first hub $k$ and a second hub $\ell$ with destination $m$,

with $j, k, \ell, m \in A$. Since we assume that any origin is allocated to itself if it is a hub, the above definition implies that site $k$ is opened as a hub if the corresponding variable $x_{k k}$ takes the value 1 .

The formulation of the model is:

$$
\begin{aligned}
\left(F_{\bar{u}}\right) \min & \sum_{i \in A} \sum_{h=2}^{G} \lambda_{i}\left(\hat{c}_{(h)}-\hat{c}_{(h-1)}\right) \bar{u}_{i h}+\sum_{k \in A} \sum_{\ell \in A} \sum_{m \in A}\left(\mu c_{k \ell}+\delta c_{\ell m}\right) s_{k \ell m} \\
\text { s.t. } & \sum_{k \in A} x_{j k}=1, \quad \forall j \in A \\
& \sum_{j \in A} x_{j k} \leq n x_{k k}, \quad \forall k \in A \\
& \sum_{k \in A} x_{k k}=p \\
& \sum_{\ell \in A} s_{k \ell m}=\sum_{j \in A} w_{j m} x_{j k}, \quad \forall k, m \in A \\
& s_{k \ell m} \leq \sum_{j \in A} w_{j m}\left(1-x_{m m}\right) \quad \forall k, \ell, m \in A, \quad \ell \neq m \\
& \sum_{\ell \in A} \sum_{m \in A} s_{k \ell m} \leq x_{k k} \sum_{j \in A} W_{j}, \quad \forall k \in A \\
& \sum_{k \in A} \sum_{m \in A} s_{k \ell m} \leq x_{\ell \ell} \sum_{j \in A} W_{j}, \quad \forall \ell \in A \\
& \sum_{j \in A} W_{j} x_{j k} \leq b_{k} x_{k k}, \quad \forall k \in A \\
& \sum_{i \in A} \bar{u}_{i h}=\sum_{j \in A} \sum_{\hat{c}_{j k} \geq \hat{c}_{(h)}}^{n} x_{j k}, \quad \forall h=1, \ldots, G \\
& \bar{u}_{i h} \geq \bar{u}_{i-1, h}, \forall i \in A \backslash\{1\}, h=1, \ldots, G \\
& \bar{u}_{i h}, x_{j k} \in\{0,1\}, s_{k \ell m} \geq 0, \quad \forall i, j, k, \ell, m \in A, \quad h=1, \ldots, G
\end{aligned}
$$

The objective function (3) accounts for the weighted sum of the three components of the shipping cost, namely origin-first hub, hub-hub and hub-destination. The origin-hub costs are 
accounted after assigning the lambda parameters, i.e. $\sum_{i \in A} \sum_{h=2}^{G} \lambda_{i} \cdot\left(\hat{c}_{(h)}-\hat{c}_{(h-1)}\right) \cdot \bar{u}_{i h}$. In addition, the second and third blocks of delivery costs, i.e. the hub-hub and hub-destination cost, scaled with the $\mu$ and $\delta$ parameters respectively, can be stated as: $\sum_{k \in A} \sum_{\ell \in A} \sum_{m \in A}\left(\mu c_{k \ell}+\right.$ $\left.\delta c_{\ell m}\right) s_{k \ell m}$.

The constraints of the model are described in [36] with the only exception of (11), that is the capacity constraint on the hubs, in spite of that and for the sake of completeness we include below a brief description of them. Constraints (4) ensure that the flow from the origin site $j$ is associated with a unique first hub. Constraints (5) ensure that any origin only can be allocated to an open hub. Constraint (6) fixes the number of hubs to be located. Constraints (77) are flow conservation constraints, such that the flow that enters any hub $k$ with final destination $m$ is the same that the flow that leaves hub $k$ with destination $m$. Constraints (8) ensure that if the final destination site is a hub, then the flow goes at most through one additional hub. These constraints are redundant whenever the cost structure satisfies the triangular inequality, however they are useful in reducing solution times (see [36]). Constraints (9) and (10) establish again that the intermediate nodes in any origin-destination path should be open hubs. Constraints (11) establish the capacity constraints of the hubs. Observe that this family of constraints make redundant the family (5), but we have kept it because it reduces the computational times. Constraints (12) link sorting and covering variables. They state that the number of allocations with a cost at least $\hat{c}_{(h)}$ must be equal to the number of sites that support shipping costs to the first hub greater than or equal to $\hat{c}_{(h)}$. Finally, constraints (13) are a group of sorting conditions on the variables $\bar{u}_{i h}$.

The reader may note that this formulation is a natural extension for the capacitated version of the radius formulation already considered for the uncapacitated ordered median hub location problem in [36, 37]. However, although this formulation is enough to specify the CSA-OMHLP, we have found that for solving medium sized problems it produces very large MIP models, which are difficult to solve with standard MIP solvers (CPLEX, XPRESS; Gurobi...). Therefore, some alternatives should be investigated.

One way to improve the performance of the above formulation is to take advantage of some features of that model to reduce the number of variables. In this case, one can suc- 
ceed reducing the number of $u$ variables. The logic of the above formulation can be further strengthen for important particular cases of the discrete ordered median hub location problem. In the following, we show a reformulation that is based on taking advantage of sequences of repetitions in the $\lambda$-vector. (See [33, 37, 38] for similar reformulations applied to other location problems.)

One can realize that for $\lambda$-vectors with sequences of repetitions -i.e. the center, $k$-centrum, trimmed means or median among others-, many variables used in formulation $F_{\bar{u}}$ are not necessary (since they are multiplied by zero in the objective function), and some others can be glued together (since they have the same coefficient in the objective function). Moreover, under the assumption of the free self-service, and that any origin is allocated to itself if it is a hub, we conclude that the $p$ smallest transportation costs from the origin to the first hubs are 0 , i.e. the first $p$ components of the $\lambda$-vector are multiplied by 0 . Therefore, in order to simplify the problem one can disregard the $p$ first components of the $\lambda$-vector. Let $\tilde{\lambda}=\left(\tilde{\lambda}_{1}, \ldots, \tilde{\lambda}_{n-p}\right):=\left(\lambda_{p+1}, \ldots, \lambda_{n}\right)$.

In order to give a formulation for the CSA-OMHLP taking advantage of these facts, we need to introduce some additional notation. Let $I$ be the number of blocks of consecutive equal non-null elements in $\tilde{\lambda}$ and define the vectors:

1. $\gamma=\left(\gamma_{1}, \ldots, \gamma_{I}\right)$, being $\gamma_{i}, i=1, \ldots, I$ the value of the elements in the $i$-th block of repeated elements in $\tilde{\lambda}$.

2. $\alpha=\left(\alpha_{1}, \ldots, \alpha_{I}, \alpha_{I+1}\right)$, being $\alpha_{i}$ with $i=1, \ldots, I$, the number of zero entries between the $(i-1)$-th and $i$-th blocks of positive elements in $\tilde{\lambda}$ and $\alpha_{I+1}$ the number of zeros, if any, after the $I$-th block of non-null elements in $\tilde{\lambda}$. For notation purposes we define $\alpha_{0}=0$.

3. $\beta=\left(\beta_{1}, \ldots, \beta_{I}\right)$, being $\beta_{i}, i=1, \ldots, I$ the number of elements in the $i$-th block of non-null elements in $\tilde{\lambda}$. For the sake of compactness, let $\beta_{0}=\beta_{I+1}=0$.

Next, let denote $\bar{\alpha}_{i}=\sum_{j=1}^{i} \alpha_{j}, \bar{\beta}_{i}=\sum_{j=1}^{i} \beta_{j}$ and recall that $W_{i}=\sum_{j \in A} w_{i j}$. Moreover, for 
all $i=1, \ldots, I$ and $h=1, \ldots, G$, let us define the following set of decision variables:

$$
\begin{aligned}
& u_{i h}= \begin{cases}1, & \text { if the }\left(p+\bar{\alpha}_{i}+\bar{\beta}_{i-1}+1\right) \text {-th assignment cost is at least } \hat{c}_{(h)}, \\
0, & \text { otherwise }\end{cases} \\
& v_{i h}=\text { Number of assignments in the } i \text {-th block between the positions } \\
& p+\bar{\alpha}_{i}+\bar{\beta}_{i-1}+1 \text { and } p+\bar{\alpha}_{i}+\bar{\beta}_{i} \text { that are at least } \hat{c}_{(h)} .
\end{aligned}
$$

With the above notation the formulation of CSA-OMHLP is:

$$
\begin{aligned}
\left(F_{u v}\right) \min & \sum_{i=1}^{I} \sum_{h=2}^{G} \gamma_{i}\left(\hat{c}_{(h)}-\hat{c}_{(h-1)}\right) v_{i h}+\sum_{k \in A} \sum_{\ell \in A} \sum_{m \in A}\left(\mu c_{k \ell}+\delta c_{\ell m}\right) s_{k \ell m} \\
\text { s.t. } \quad \text { Constraints }:(41)-(11), & \\
& \sum_{i=1}^{I} \alpha_{i} u_{i h}+\sum_{i=1}^{I} v_{i h}+\alpha_{I+1} \geq \sum_{j \in A} \sum_{\substack{\hat{c}_{j k} \geq A \\
\hat{c}_{(h)}}} x_{j k}, \quad \forall h=2, \ldots, G \\
& u_{i h} \geq u_{i-1, h}, \quad \forall i=2, \ldots, I, h=1, \ldots, G \\
& \beta_{i-1} u_{i h} \geq v_{i-1, h}, \quad \forall i=2, \ldots, I, h=1, \ldots, G \\
& v_{i h} \geq \beta_{i} u_{i h}, \quad \forall i=1, \ldots, I, \quad h=1, \ldots, G \\
& u_{i h} \in\{0,1\}, v_{i h} \in \mathbb{Z} \cap\left[0, \beta_{i}\right], \quad \forall i=1 \ldots, I, \quad h=1, \ldots, G \\
& x_{j k} \in\{0,1\}, s_{k \ell m} \geq 0, \quad \forall j, k, \ell, m \in A .
\end{aligned}
$$

The objective function (15) is a reformulation of (3) substituting the $\bar{u}$ variables by the new $u, v$ variables and the vector $\gamma$, taking advantage of the $\lambda$ vector properties. Constraints (16) ensure that the number of sites that support a shipping cost to the first hub greater than or equal to $\hat{c}_{(h)}$ is either equal to the number of allocations with a cost at least $\hat{c}_{(h)}$ whenever $v_{I h}>0$ or less than or equal to $\alpha_{I+1}$ otherwise. Constraints (17) are sorting constraints on the variables $u$ similar to constraints (13), and constraints (18)-(19) provide upper and lower bounds on the variables $v$ depending on the values of variables $u$.

The main difference between $F_{\bar{u}}$ and $F_{u v}$ is that all $\bar{u}_{i h}$ variables associated with blocks of zero $\lambda$-values are removed, and those associated with each block of non-null $\lambda$ values are replaced by $2 \times G$ variables. Therefore, overall we reduce the number of variables by $(n-2 I) \times G$. 
Note that in Formulation $F_{\bar{u}}$, the family of constraints that links covering variables (variables $\bar{u}$ ) and the allocation variables (variables $x$ ), i.e. (12), is given with equalities. This fact implies that the actual dimension of the feasible region in the space of $\bar{u}$ and $x$ variables is smaller than the one that we were currently working on. This is exploited in the new formulation. Indeed, we reduce the number of variables used in the sorting phase replacing $\bar{u}$ by $u$ and $v$. Therefore, the dimension of the feasible region in the space of $u, v, x$ variables has smaller dimension. In addition, the constraints that link sorting and design variables, namely (16), are given as inequalities. This new representation, although valid for the problem, induces some loss of information in that it does not allow us to take full control of the exact number of allocations at some specific cost. This does not affect the resolution process but influences the derivation of valid inequalities.

Finally, for those cases where $\beta_{i}=1$ we observe that $v_{i h}=u_{i h}$. This set of constraints whenever valid, was added to reinforce the formulation.

Example 2.1 To illustrate how the $F_{u v}$ versus $F_{\bar{u}}$ formulations work, we consider the following data. Let $A=\{1, \ldots, 6\}$ be a set of sites and assume that we are interested in locating $p=2$ hubs. Let the cost and flow matrices be as follows:

$$
C=\left(\begin{array}{cccccc}
0 & 14 & 15 & 16 & 15 & 9 \\
5 & 0 & 7 & 2 & 19 & 16 \\
16 & 5 & 0 & 7 & 1 & 19 \\
12 & 1 & 10 & 0 & 13 & 1 \\
1 & 9 & 9 & 15 & 0 & 2 \\
8 & 10 & 16 & 8 & 4 & 0
\end{array}\right), W=\left(\begin{array}{cccccc}
0 & 15 & 2 & 8 & 11 & 2 \\
19 & 0 & 1 & 16 & 20 & 7 \\
3 & 9 & 0 & 3 & 11 & 16 \\
7 & 2 & 5 & 0 & 14 & 5 \\
15 & 4 & 20 & 4 & 0 & 1 \\
12 & 4 & 7 & 11 & 18 & 0
\end{array}\right) .
$$

Therefore, $\hat{c}_{(\cdot)}$, the sorted vector of $\hat{c}$, is in our case

$\hat{c}_{(\cdot)}=[0,33,42,44,88,126,208,210,294,315,330,342,396,416,429,441,520,532,570,608,660$, $672,798,832,1008,1197]$. Hence, $G=26$. Let $\lambda=(0,1,0,0,1,1), \mu=0.7, \delta=0.9$, and the capacity constraints vector $b=(119,119,113,145,149,140)$. The optimal solution opens hubs 4 and 6. The allocation of origin sites to first hub is given by the following values of the variables $x$ (see Figure 1):

$$
x_{16}=x_{24}=x_{34}=x_{44}=x_{56}=x_{66}=1 .
$$

Analogously, the allocation of first hubs to final destinations are given by the values of the non null variables s. Thus, the flows considering as first hubs 4 and 6 are (see Figure 1 for a 
graphical representation of the delivery paths):

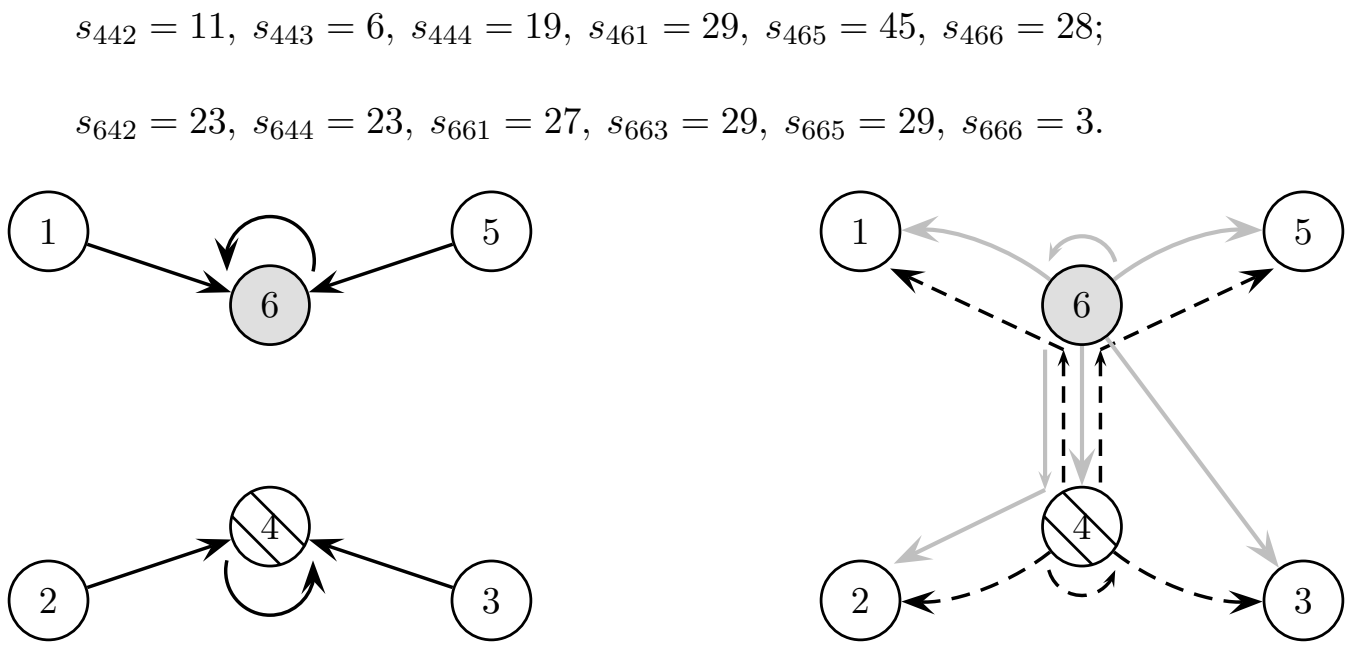

Figure 1: Illustration of Example 2.1. Left figure represents the allocations of sites to their corresponding first hubs. Right figure represents the flow pattern to the final destinations from the first hubs: 4 (dashed lines) and 6 (grey lines).

Moreover, the covering variables $\bar{u}_{i h}$ are given below. Due to their structure, we only report for each $i$ the last one and first zero occurrences since they characterize the remaining values.

$$
\begin{array}{lll}
i=1 \mapsto \bar{u}_{11}=1, \bar{u}_{12}=0 & i=2 \mapsto \bar{u}_{21}=1, \bar{u}_{22}=0 & i=3 \mapsto \bar{u}_{35}=1, \bar{u}_{36}=0 \\
i=4 \mapsto \bar{u}_{46}=1, \bar{u}_{47}=0 & i=5 \mapsto \bar{u}_{59}=1, \bar{u}_{5,10}=0 & i=6 \mapsto \bar{u}_{6,12}=1, \bar{u}_{6,13}=0 .
\end{array}
$$

The first two assignments are done at a cost $c_{(1)}=0$, corresponding to the two hubs $(p=2)$. The next assignment has been done at a cost $c_{(5)}=88$, since $\bar{u}_{35}=1$ and $\bar{u}_{36}=0$, and so on. The rest of assignments costs are then $c_{(6)}=126, c_{(9)}=294$ and $c_{(12)}=342$.

Hence, the overall cost of this solution is

$$
\sum_{i \in A} \sum_{h=2}^{G} \lambda_{i}\left(\hat{c}_{(h)}-\hat{c}_{(h-1)}\right) \bar{u}_{i h}+\sum_{k \in A} \sum_{\ell \in A} \sum_{m \in A}\left(\mu c_{k \ell}+\delta c_{\ell m}\right) s_{k \ell m}=636+1500.8=2136.8 .
$$

In addition, to illustrate how the formulation $F_{u v}$ is related with $F_{\bar{u}}$, we also include the solution of the covering variables $u_{i h}$ and $v_{i h}$ :

$$
\begin{aligned}
& I=1 \mapsto u_{16}=1, u_{17}=0 \\
& I=1 \mapsto v_{11}=\ldots=v_{19}=2 ; v_{1,10}=\ldots=v_{1,12}=1 ; v_{1,13}=0
\end{aligned}
$$




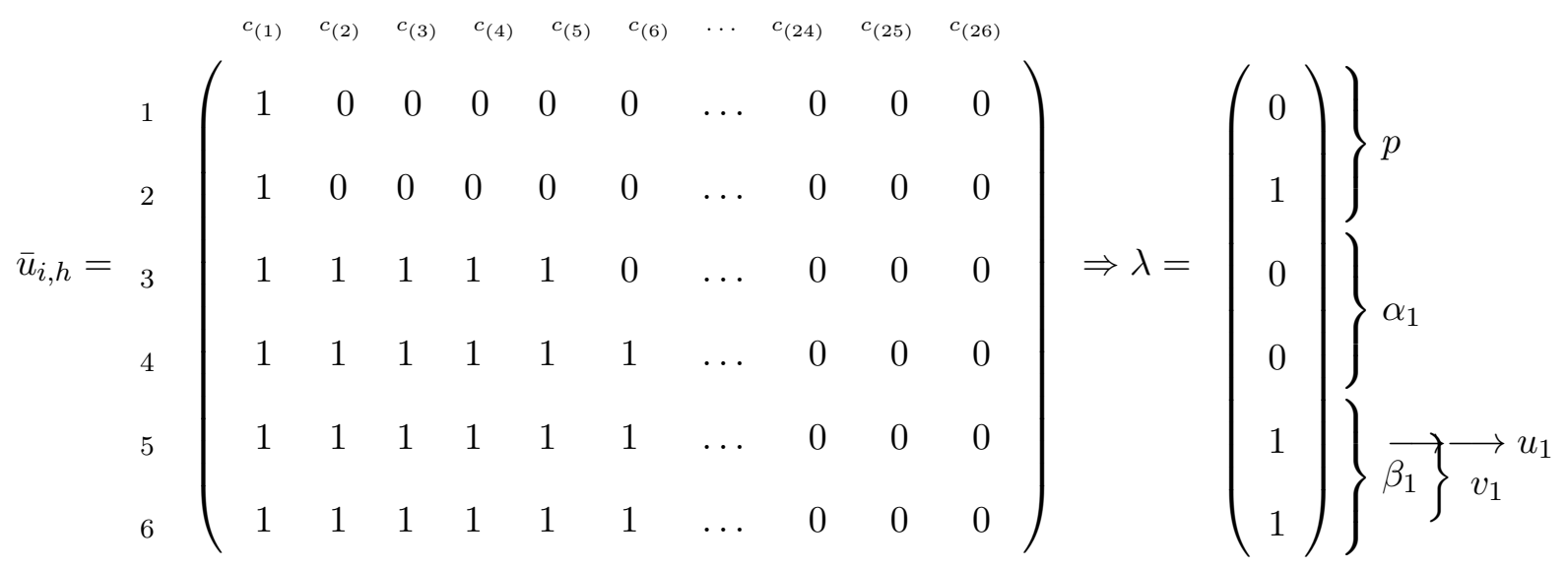

Figure 2: Variables and lambda vector of Example 2.1

Note that we have only one block of repeated non-null elements of the $\tilde{\lambda}$-vector, so $I=1$. (See the right part of Figure Q.) The number of zero entries between two blocks is $\alpha_{1}=2$, and the number of elements in the 1 -st block of non-null elements is $\beta_{1}=2$. Furthermore, $\gamma_{1}=1$ is the repeated value in the 1-st block.

$$
\begin{aligned}
& \begin{array}{llllllllllllllll}
c_{(1)} & c_{(2)} & c_{(3)} & \ldots & c_{(9)} & c_{(10)} & c_{(11)} & c_{(12)} & c_{(13)} & c_{(14)} & c_{(15)} & c_{(16)} & c_{(17)} & \ldots & c_{(25)} & c_{(26)}
\end{array} \\
& u_{1, h}=\left(\begin{array}{ccccccccccccccccc}
1 & 1 & 1 & \ldots & 1 & 1 & 1 & 1 & 1 & 1 & 1 & 1 & 0 & \ldots & 0 & 0
\end{array}\right) \\
& v_{1, h}=\left(\begin{array}{ccccccccccccccccc}
2 & 2 & 2 & \ldots & 2 & 1 & 1 & 1 & 0 & 0 & 0 & 0 & 0 & \ldots & 0 & 0
\end{array}\right)
\end{aligned}
$$

The variable $u_{1, h}$ points out to the row $p+\alpha_{1}+1=5$ of the original variable $\bar{u}_{i, h}$. Whereas the variable $v_{1, h}$ accounts for the number of assignments between the positions $p+\alpha_{1}+1=5$ and $p+\alpha_{1}+\beta_{1}=6$ of $\bar{u}_{i, h}$ that are at least $\hat{c}_{(h)}$. (See Figure Q .)

From $\bar{u}_{i, h}$, we know that the 5 -th assignment cost is $\hat{c}_{(9)}$ and the 6 -th assignment cost is $\hat{c}_{(12)}$. For this reason $v_{1, h}$ is equal to 2 up to column 9 , this is the number of assignment costs greater than $\hat{c}_{(9)}$. Being this number equal to 1 from $h=10$ to $h=12$, and zero for the remaining columns.

Applying this formulation, the overall reduction in the number of variables is $(n-2 I) \times G=$ 104. The rest of variables $x$ and s remain the same, and again the overall cost of this solution 


$$
\sum_{i=1}^{I} \sum_{h=2}^{G} \gamma_{i}\left(\hat{c}_{(h)}-\hat{c}_{(h-1)}\right) v_{i h}+\sum_{k \in A} \sum_{\ell \in A} \sum_{m \in A}\left(\mu c_{k \ell}+\delta c_{\ell m}\right) s_{k \ell m}=636+1500.8=2136.8
$$

\section{Strengthening the formulation}

\subsection{Variable fixing}

Next, we describe some preprocessing procedures that we have applied to reduce further the size of formulation $F_{u v}$. We present a number of variable fixing possibilities for the set of variables $u$ and $v$ which are useful in the overall solution process. The variable fixing procedures developed in this section are based on ideas used in [36, 37] and taking advantage of the capacity constraints. Indeed, we are adding the reinforced effective capacity constraints, as well as some surrogated version of constraints (24) since in this aggregated form they give better running times. The preprocessing phase developed in this paper also provides new upper and lower bounds on the $v$ variables. The percentage of variable reduction obtained by these procedures can be found in Tables 1 and 2 (column named as 'Fixed').

Before describing these procedures for fixing variables, the following simple arguments allows us to fix some variables:

1. First, $c_{j j}=0 \forall j \in A$, i.e., $\hat{c}_{(1)}=0$. Moreover, every origin where it has been located a hub will be allocated to itself as a first hub.

2. Second, $\hat{c}_{j k} \neq 0$ if and only if $j \neq k$, i.e., any non-hub origin is allocated to a first hub at a cost of at least $\hat{c}_{(2)}$.

Therefore, since in this formulation the first $p$-allocations are considered only implicitly, we can fix $u_{i 1}=1, v_{i 1}=\beta_{i}$ as well as $u_{i 2}=1, v_{i 2}=\beta_{i}, \forall i=1, \ldots, I$.

\subsubsection{Preprocessing Phase 1: Fixing variables to the upper bounds for the formulation with covering variables strengthen with capacity constraints.}

Due to the definition of the variables in formulation $F_{u v}$, one can expect that $u_{i h}=1$ whenever $i$ is large and $h$ is small to medium size because this would mean that the $\left(p+\bar{\alpha}_{i}+\bar{\beta}_{i-1}+1\right)$ - 
th sorted allocation cost would not have been done at cost less than $\hat{c}_{(h)}$. The reader should observe that an analogous strategy applies to the variables $v$ since their interpretation is similar, but in this case the values of $v_{i h}$ would be fixed to $\beta_{i}$. For the cases where it is not possible to fix the corresponding $v$-variable, it could be still possible to establish some lower bounds as we will see later.

Next, to fix variables $u_{i h}$ and $v_{i h}$ for $i=1, \ldots, I, h=1, \ldots, G$, we deal with an auxiliary problem that maximizes the number of variables that may assume zero values, satisfying $\hat{c}_{j k} \leq \hat{c}_{(h-1)}$ and the capacity constraints. For any $h=1, \ldots, G$ and $j, k \in A$ such that $\hat{c}_{j k} \leq \hat{c}_{(h-1)}$ let

$$
z_{j k}^{h}= \begin{cases}1, & \text { if origin site } j \text { is assigned to hub } k \\ 0, & \text { otherwise }\end{cases}
$$

To avoid possible misunderstanding in the cases where variables $z_{j k}^{h}$ are not defined, i.e. when $\hat{c}_{j k}>\hat{c}_{(h-1)}$, we can assume that $z_{j k}^{h}:=0$.

For a given $h$, we introduce the effective capacity of a hub $k$ at a cost at most $\hat{c}_{(h-1)}$ as,

$$
b_{k}^{h-1}:=\min \left\{b_{k}, \sum_{\substack{s \in A \\ \hat{c}_{s k} \leq \hat{c}_{(h-1)}}} W_{s}\right\} .
$$

Indeed, the capacity of a hub $k$ is always lower than or equal to $b_{k}$. In addition, if we restrict ourselves to the nodes served at a cost of at most $\hat{c}_{(h-1)}$, then the actual capacity to cover this set should be lower than $\sum_{s \in A: \hat{c}_{s k} \leq \hat{c}_{(h-1)}} W_{s}$, and this gives us the expression of $b_{k}^{h-1}$.

The optimal value $P_{1}(h)$ of the following problem fixes the maximal number of allocations that may be feasible at a cost of at most $\hat{c}_{(h-1)}$.

$$
\begin{aligned}
P_{1}(h):=\max & \sum_{\substack{j, k \in A: \\
\hat{c}_{j k} \leq \hat{c}_{(h-1)}}} z_{j k}^{h} \\
\text { s.t. } & \sum_{\substack{k \in A: \hat{c}_{j k} \leq \hat{c}_{(h-1)} \\
j \in A: \hat{c}_{j k} \leq \hat{c}_{(h-1)}}} z_{j k}^{h} \leq 1, \quad \forall j \in A, \\
& \sum_{k \in A} y_{k} \leq p
\end{aligned}
$$




$$
\begin{gathered}
\sum_{j \in A: \hat{c}_{j k} \leq \hat{c}_{(h-1)}} W_{j} z_{j k}^{h} \leq b_{k}^{h-1} y_{k}, \quad \forall k \in A \\
z_{j k}^{h}, y_{k} \in\{0,1\}, \quad \forall j, k \in A, \forall h=1, \ldots, G .
\end{gathered}
$$

Then, depending on the value $P_{1}(h)$ we can fix some variables to their upper bounds. Let us denote by $i_{1}(h) \in\{1, \ldots, I\}$ the index such that

$$
\begin{gathered}
p+\bar{\alpha}_{i_{1}(h)-1}+\bar{\beta}_{i_{1}(h)-1}<P_{1}(h) \leq p+\bar{\alpha}_{i_{1}(h)}+\bar{\beta}_{i_{1}(h)} . \\
\begin{cases}u_{i h}=1, v_{i h}=\beta_{i}, i=i_{1}(h), \ldots, I, & \text { if } P_{1}(h) \leq p+\bar{\alpha}_{i_{1}(h)}+\bar{\beta}_{i_{1}(h)-1} \\
\left\{\begin{array}{l}
v_{i_{1}(h), h} \geq p+\bar{\alpha}_{i_{1}(h)}+\bar{\beta}_{i_{1}(h)}-P_{1}(h), \\
u_{i h}=1, \quad v_{i h}=\beta_{i}, i=i_{1}(h)+1, \ldots, I,
\end{array}\right\} & \text { otherwise. }\end{cases}
\end{gathered}
$$

\subsubsection{Preprocessing Phase 2: Fixing variables to their lower bounds}

Following similar argument to the previous subsection, one can expect that many variables $u$ and $v$ in the top-right hand corner of the matrices of variables $u$ and $v$, respectively, will take value 0 in the optimal solution. Indeed, $u_{i h}=0$ means that the $\left(p+\bar{\alpha}_{i}+\bar{\beta}_{i-1}+1\right)$-th sorted allocation cost is less than $\hat{c}_{(h)}$ which is very likely to be true if $h$ is sufficiently large and $i$ is small. Note that an analogous strategy, applies to the variables $v$ since their interpretation is similar. For the cases where it is not possible to fix the corresponding $v$-variable it could be still possible to establish some upper bounds.

For any $h=2, \ldots, G, j, k \in A$ such that $\hat{c}_{j k} \geq \hat{c}_{(h-1)}$ let define variables $z_{j k}^{h}$ as (22). To avoid possible misunderstanding in the cases where variables $z_{j k}^{k}$ are not defined, i.e. when $\hat{c}_{j k}<\hat{c}_{(h-1)}$, we can assume that $z_{j k}^{h}:=0$. Using these variables, the formulation of the problem that maximizes the number of non-fixed allocations at a cost at most $\hat{c}_{(h-1)}$ is:

$$
\begin{aligned}
P_{2}(h):=\max & \sum_{\substack{j, k \in A: \\
\hat{c}_{j k} \geq \hat{c}_{(h-1)}}} z_{j k}^{h} \\
\text { s.t. } & \sum_{k \in A: \hat{c}_{j k} \geq \hat{c}_{(h-1)}} z_{j k}^{h} \leq 1, \quad \forall j \in A \\
& \sum_{j \in A: \hat{c}_{j k} \geq \hat{c}_{(h-1)}} z_{j k}^{h} \leq n y_{k}, \quad \forall j, k \in A
\end{aligned}
$$




$$
\begin{aligned}
& \sum_{k \in A} y_{k} \leq p \\
& z_{j k}^{h}, y_{k} \in\{0,1\}, \quad \forall j, k \in A, \forall h=1, \ldots, G .
\end{aligned}
$$

Note that the value $P_{2}(h)$ implies that there are no feasible solutions of the original problem with less than $n-P_{2}(h)$ allocations fixed at a cost at most $\hat{c}_{(h)}$.

Let $1 \leq i_{2}(h) \leq I$ be the index such that

$$
p+\bar{\alpha}_{i_{2}(h)-1}+\bar{\beta}_{i_{2}(h)-1}<n-P_{2}(h) \leq p+\bar{\alpha}_{i_{2}(h)}+\bar{\beta}_{i_{2}(h)} .
$$

Thus, in any feasible solution of the problem we have that:

$$
\begin{cases}u_{i h}=0, v_{i h}=0, i=1, \ldots, i_{2}(h)-1, & \text { if } n-P_{2}(h) \leq p+\bar{\alpha}_{i_{2}(h)}+\bar{\beta}_{i_{2}(h)-1} \\
\left\{\begin{array}{l}
u_{i_{2}(h), h}=0, \quad v_{i_{2}(h), h} \leq p+\bar{\alpha}_{i_{2}(h)}+\bar{\beta}_{i_{2}(h)}-\left(n-P_{2}(h)\right), \\
u_{i h}=0, \quad v_{i h}=0, \quad i=1, \ldots, i_{2}(h)-1,
\end{array}\right\} & \text { otherwise. }\end{cases}
$$

Note that whenever $n-P_{2}(h)=p$ then there is nothing to fix and therefore no variables are set to zero in column $h$.

\subsection{Valid Inequalities}

In order to strengthen formulation $F_{u v}$ we have studied several families of valid inequalities. In fact, taking advantage of previous experience on the non-capacitated version of the problem we have borrowed a first family of valid inequalities that are very simple and that have proven to be effective in different ordered median problems with covering variables [36, 37]. This family is

$$
\begin{aligned}
& u_{i h} \geq u_{i, h+1}, \quad i=1, \ldots, I, h=1, \ldots, G-1 \\
& v_{i h} \geq v_{i, h+1}, \quad i=1, \ldots, I, h=1, \ldots, G-1 .
\end{aligned}
$$

Since, these families are straightforward consequence of the definition of variables $u$ and $v$ they have been included in the original formulation.

In the following, we describe several alternative families of valid inequalities: three sets of inequalities, (28), (29)-(32), and (33)-(36), based on the combination of ordering and capacity requirements and two more sets, (37) and (38)-(39), that do not use capacities. 


\subsubsection{First family of valid inequalities: Valid inequalities based on capacity I}

We can add to this model several families of valid inequalities based on capacity issues that help in solving the problem by reducing the gap of the linear relaxation and the CPU time to explore the branch and bound search tree.

Observe that the capacity of the set of hubs that may be used to assign origins in $A$ at a cost at most $\hat{c}_{(h-1)}$, is given by

$$
\sum_{k \in A} b_{k}^{h-1} x_{k k}
$$

where $b_{k}^{h-1}$ is the effective capacity at a cost at most $\hat{c}_{(h-1)}$, defined by (23). Recall that, although the capacity of a hub $k$ is always lower than or equal to $b_{k}$, when we restrict to the nodes served at a cost of at most $\hat{c}_{(h-1)}$, then the actual capacity to cover this set should be lower than $b_{k}^{h-1}$. Making use of the above observation, we can add the following family of constrains as valid inequalities

$$
\sum_{\substack{j \in A \\ \hat{c}_{j k} \leq \hat{c}_{(h-1)}}} W_{j} x_{j k} \leq b_{k}^{h-1} x_{k k} \quad \forall h=2, \ldots, G, k \in A
$$

which enforces that all the flow sent from origin-hubs at a cost at most $\hat{c}_{(h-1)}$ cannot exceed the effective capacity at that cost. Observe that in the case where $b_{k}^{h-1}$ takes the value $b_{k}$ (11) dominates (28), but in the case where $b_{k}^{h-1}=\sum_{s \in A: \hat{c}_{s k} \leq \hat{c}_{(h-1)}} W_{s}$, (28) becomes $\sum_{j \in A: \hat{c}_{j k} \leq \hat{c}_{(h-1)}} W_{j} x_{j k} \leq \sum_{j \in A: \hat{c}_{j k} \leq \hat{c}_{(h-1)}} W_{j} x_{k k}$. Observe that this last valid inequality is an alternative surrogation, with capacity coefficients, of constraints $x_{j k} \leq x_{k k}$ that although valid do not appear in the model because of its large cardinality. This new form of aggregation has provided good results in the computational experiments.

\subsubsection{Second family of valid inequalities: Valid inequalities based on capacity II}

This section introduces another family of valid inequalities based on capacity issues that help in solving the problem. In order to present these new valid inequalities based on capacity requirements we introduce some new notation. Assume without loss of generality that $W_{i} \leq$ $W_{i+1}$ for $i \in A \backslash\{n\}$ and let $\bar{W}_{j}=\sum_{r=1}^{j} W_{r}$ and $S_{k}:=\{i \in A: i \leq k\}$ for $k \in A$ be a given set of origin sites. 
In case that the effective capacity at a cost at most $\hat{c}_{(h-1)}$ is not sufficient to cover the demand of $S_{k}$, i.e. $\sum_{j=1}^{n}\left(b_{j}^{h-1}-W_{j}\right) x_{j j}$ is less than $\sum_{s=1}^{k} W_{s}\left(1-x_{s s}\right)$, then at most $k-1$ origins of $S_{k}$ can be allocated at a cost lower than or equal to $\hat{c}_{(h-1)}$. This argument can be applied for each $h$ to the corresponding $\hat{c}_{(h-1)}$ value. Moreover, we have chosen this particular structure of $S_{k}$ consisting of the $k$ origins (nodes) with the $k$-smallest flows, because given a fixed amount of flow, the maximal cardinality set of origins, such that the overall flow originated in this set is lower than or equal to this amount, is provided by a set $S_{k}$ for some $k \in A$. Therefore, since we are dealing with the worst cases, it allows us to fix some variables $u$ and $v$ through the following valid inequalities. For each $h=2, \ldots, G$ we obtain the following:

- If $\bar{\alpha}_{i-1}+\bar{\beta}_{i-1}<k \leq \bar{\alpha}_{i}+\bar{\beta}_{i-1}+1$, for some $i \in\{1, \ldots, I\}$, namely if the index of the last element, $k$, that defines $S_{k}$ lies in the $i$-th block of null elements in the $\tilde{\lambda}$ vector, then

$$
\bar{W}_{k} u_{i h}+\sum_{j \in A}\left(b_{j}^{h-1}-W_{j}\right) x_{j j} \geq \sum_{s=1}^{k} W_{s}\left(1-x_{s s}\right) .
$$

Observe that the above inequality amounts to a disjunctive condition: either the effective capacity at a cost at most $\hat{c}_{(h-1)}$ is enough to cover the demand of the $k$ smallest flows from origin sites or the $i$-th sorted cost allocation must be assigned at a cost at least $c_{(h)}$.

- If $\bar{\alpha}_{i}+\bar{\beta}_{i-1}+1<k \leq \bar{\alpha}_{i}+\bar{\beta}_{i}$, and namely if the index of the last element, $k$, that defines $S_{k}$ lies in the $i$-th block of non-null elements, then

$$
\bar{W}_{k} v_{i h}+\sum_{j \in A}\left(b_{j}^{h-1}-W_{j}\right) x_{j j} \geq \sum_{s=1}^{k} W_{s}\left(1-x_{s s}\right) .
$$

In this case, the inequality is similar to the previous one but written in terms of the variables $v$ that allow to control the capacity whenever $k$ falls within a block of non-null elements in the $\tilde{\lambda}$ vector.

Remark 3.1 Recall that if $k>P_{1}(h)-p$, variables $u_{i h}$ and $v_{i h}$ have been already fixed by the Preprocessing Phase 1, and for the above inequality to be effective $k \leq P_{1}(h)-p$, or equivalently, $i \leq i_{1}(h)$. 
Based on the same arguments we can add a larger family of valid inequalities built on arbitrary sets of origin sites. Let $S$ be a set of origin sites, and suppose that $A_{S}=\sum_{s \in S} W_{s}$ satisfies $\bar{W}_{k} \leq A_{S}<\bar{W}_{k+1}$,

- If $\bar{\alpha}_{i-1}+\bar{\beta}_{i-1}<k \leq \bar{\alpha}_{i}+\bar{\beta}_{i-1}+1$, for some $i \in\{1, \ldots, I\}$, and $k \leq P_{1}(h)-p$ then

$$
A_{S} u_{i h}+\sum_{j \in A}\left(b_{j}^{h-1}-W_{j}\right) x_{j j} \geq \sum_{s=1}^{k} W_{s}\left(1-x_{s s}\right) .
$$

- If $\bar{\alpha}_{i}+\bar{\beta}_{i-1}+1<k \leq \bar{\alpha}_{i}+\bar{\beta}_{i}$, and $k \leq P_{1}(h)-p$ then

$$
A_{S} v_{i h}+\sum_{j \in A}\left(b_{j}^{h-1}-W_{j}\right) x_{j j} \geq \sum_{s=1}^{k} W_{s}\left(1-x_{s s}\right) .
$$

Now, assuming a more general case and for an improvement of the above inequalities (29) and (30), for a given $h \in\{2, \ldots, G\}$, and $\left.k \leq P_{1}(h)-p, k \in\left\{1, \ldots, \bar{\alpha}_{I+1}+\bar{\beta}_{I}\right)\right\}$, let

$$
M_{s}:=\min _{j(\neq s) \in A} \hat{c}_{j s}
$$

$M_{s}$ is the minimum allocation cost to $s$ as an open hub. In other words, no allocation to hub $s$ is possible at a cost less than $M_{s}$, except in case $s$ were a hub itself. We shall call this value the empty radius of $s$.

Define $s(h-1, k)$ to be the index of the sorted sequence of elements $W_{s}$ such that there are exactly $k$ elements $W_{s}$ with $s \leq s(h-1, k)$ and $M_{s} \leq \hat{c}_{(h-1)}$, namely $s(h-1, k)$, is the index such that

$$
\left|\left\{s: s \leq s(h-1, k) ; M_{s} \leq \hat{c}_{(h-1)}\right\}\right|=k .
$$

Then it holds that,

- If $\bar{\alpha}_{i-1}+\bar{\beta}_{i-1}<k \leq \bar{\alpha}_{i}+\bar{\beta}_{i-1}+1$, for some $i \in\{1, \ldots, I\}$, and $k \leq P_{1}(h)-p$

$$
\begin{aligned}
& \left(\bar{W}_{s(h-1, k)}-\sum_{\substack{s=1 \\
M_{s}>\hat{c}_{(h-1)}}}^{s(h-1, k)} W_{s}\right) u_{i h}+\sum_{j \in A}\left(b_{j}^{h-1}-W_{j}\right) x_{j j} \geq \\
& \sum_{\substack{s=1 \\
M_{s} \leq \hat{c}_{(h-1)}}}^{s(h-1, k)} W_{s}\left(1-x_{s s}\right)-\sum_{\substack{s=1 \\
M_{s}>\hat{c}_{(h-1)}}}^{s(h-1, k)}\left(W_{s(h-1, k)}-W_{s}\right) x_{s s} .
\end{aligned}
$$


The above inequality is also a disjunctive condition that "reinforces" the family of valid inequalities (29). It states that if the effective capacity at a cost at most $\hat{c}_{(h-1)}$ is not enough to cover the flow sent from origin sites that are not hubs and that can be allocated at some costs less than or equal to $\hat{c}_{(h-1)}$ then some of the origin sites with allocation costs less than $\hat{c}_{(h-1)}$ must be assigned at a cost at least $\hat{c}_{(h)}$. We observe that the use of $u$ variables in the left-hand side of the inequality is due to the fact that $k$ falls within a block of null elements in the $\tilde{\lambda}$ vector. A similar inequality also holds when $k$ falls within a block of non-null elements as shown below.

- If $\bar{\alpha}_{i}+\bar{\beta}_{i-1}+1<k \leq \bar{\alpha}_{i}+\bar{\beta}_{i}$, and $k \leq P_{1}(h)-p$ then

$$
\begin{aligned}
& \left(\bar{W}_{s(h-1, k)}-\sum_{\substack{s=1 \\
M_{s}>\hat{c}_{(h-1)}}}^{s(h-1, k)} W_{s}\right) v_{i h}+\sum_{j \in A}\left(b_{j}^{h-1}-W_{j}\right) x_{j j} \geq \\
& \sum_{\substack{s=1 \\
M_{s} \leq \hat{c}_{(h-1)}}}^{s(h-1, k)} W_{s}\left(1-x_{s s}\right)-\sum_{\substack{s=1 \\
M_{s}>\hat{c}_{(h-1)}}}^{s(h-1, k)}\left(W_{s(h-1, k)}-W_{s}\right) x_{s s} .
\end{aligned}
$$

This inequality is similar to the previous one whenever the index $k$ falls within a block of non-null elements in the $\tilde{\lambda}$ vector.

Finally, as the index $s(h-1, k)$ should be greater than or equal to $k$, we can split the above equations, (33) and (34), into $s(h-1, k)-k$ equivalent inequalities. This is, for any $t=k, \ldots, s(h-1, k)$, define $\hat{s}(h-1, k, t)$ to be the index of the sorted sequence of elements $W_{s}$ such that

$$
\left|\left\{s ; s \leq \hat{s}(h-1, k, t) ; M_{s} \leq \hat{c}_{(h-1)}\right\}\right|+t-\hat{s}(h-1, k, t)=k .
$$

Then it holds that,

- If $\bar{\alpha}_{i-1}+\bar{\beta}_{i-1}<k \leq \bar{\alpha}_{i}+\bar{\beta}_{i-1}+1$, for some $i \in\{1, \ldots, I\}$, and $k \leq P_{1}(h)-p$

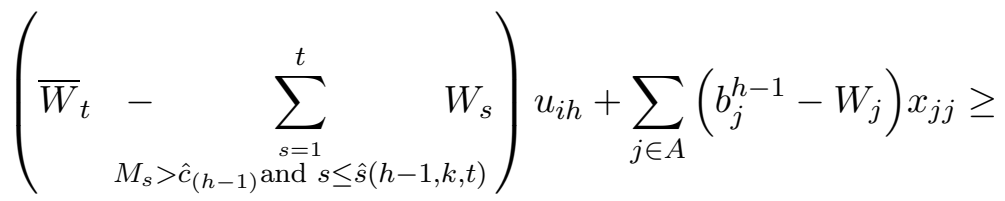

$$
\begin{aligned}
& \sum_{\substack{s=1 \\
M_{s} \leq \hat{c}_{(h-1)} \text { or } s>\hat{s}(h-1, k, t)}}^{t} W_{s}\left(1-x_{s s}\right)-\sum_{\substack{s=1 \\
M_{s}>\hat{c}_{(h-1)} \text { and } s \leq \hat{s}(h-1, k, t)}}^{t}\left(W_{t}-W_{s}\right) x_{s s} .
\end{aligned}
$$


- If $\bar{\alpha}_{i}+\bar{\beta}_{i-1}+1<k \leq \bar{\alpha}_{i}+\bar{\beta}_{i}$ and $k \leq P_{1}(h)-p$, then

$$
\begin{aligned}
& \left(\begin{array}{ccc}
\bar{W}_{t} & - & \sum_{\substack{s=1 \\
M_{s}>\hat{c}_{(h-1)}, s \leq \hat{s}(h-1, k, t)}}^{t} W_{s}
\end{array}\right) v_{i h}+\sum_{j \in A}\left(b_{j}^{h-1}-W_{j}\right) x_{j j} \geq \\
& \sum_{\substack{s=1 \\
\text { or } \\
M_{s} \leq \hat{c}(h-1, k, t)}}^{t} W_{s}\left(1-x_{s s}\right)-\sum_{\substack{s=1 \\
M_{s}>\hat{c}_{(h-1)}, s \leq \hat{s}(h-1, k, t)}}^{t}\left(W_{t}-W_{s}\right) x_{s s} .
\end{aligned}
$$

Observe that if $t=s(h-1, k)$ then $\hat{s}(h-1, k, t)=s(h-1, k)$. Thus, the families of valid inequalities (35) and (36) include as particular instances the families (33) and (34).

\subsubsection{Third family of valid inequalities: Disjunctive implications}

The third family of valid inequalities, directly borrowed from [37, state disjunctive implications on the origin-first hub allocation costs. They ensure that either origin site $j$ is allocated to a first hub at a cost of at least $\hat{c}_{(h)}$ or there is an open hub $k$ such that $\hat{c}_{j k}<\hat{c}_{(h)}$. This argument can be formulated through the following family of valid inequalities:

$$
\sum_{k \in A: \hat{c}_{j k} \geq \hat{c}_{(h)}} x_{j k}+\sum_{k \in A: \hat{c}_{j k}<\hat{c}_{(h)}} x_{k k} \geq 1, \quad \forall j \in A, h=1, \ldots, G .
$$

\subsubsection{Fourth family of valid inequalities}

Using the definition of the variables $u$ and $v$, we establish a lower and an upper bound of the number of feasible allocations at a cost $\hat{c}_{(h-1)}$. Observe that using the family of constraints (12) for the original formulation, the exact number of allocations done at a cost $\hat{c}_{(h-1)}$ is given by $\sum_{i \in A}\left(\bar{u}_{i, h-1}-\bar{u}_{i, h}\right)$. However, since in formulation $F_{u v}$ the number of variables has been considerably reduced, some information is lost. In particular, we cannot keep under control with this new formulation the exact number of allocations at a cost $\hat{c}_{(h-1)}$. Indeed, the counterpart to equalities (12) in formulation $F_{u v}$ is the family of constraints (16). Therefore, we are only able to give a lower and upper bound of this number of allocations. These lower 
and upper bounds are formulated, respectively, by the following two families of constraints:

$$
\begin{gathered}
\sum_{j \in A} \sum_{\substack{k \in A \\
\hat{c}_{j k}=\hat{c}(h-1)}} x_{j k} \geq \sum_{i=1}^{I}\left(v_{i, h-1}-v_{i h}\right)+\sum_{i=2}^{I} \alpha_{i}\left(u_{i-1, h-1}-u_{i h}\right), \quad \forall h=2, \ldots, G, \\
\sum_{j \in A} \sum_{\substack{k \in A \\
\hat{c}_{j k}=\hat{c}(h-1)}} x_{j k} \leq \sum_{i=1}^{I}\left(v_{i, h-1}-v_{i, h}\right)+\sum_{i=1}^{I} \alpha_{i}\left(u_{i, h-1}-u_{i, h}\right)+\left(1-u_{I h}\right) \alpha_{I+1}+ \\
\alpha_{1} u_{1 h}+\sum_{i=1}^{I-1} \alpha_{i+1}\left(u_{i+1, h}-u_{i h}\right), \quad \forall h=2, \ldots, G .
\end{gathered}
$$

The first sum in the right hand side of both families gives the exact number of allocations at cost $\hat{c}_{(h-1)}$ in the positions corresponding to non-null blocks of the vector $\lambda$. However, the second sum in the right hand side of constraints (38) provides a lower bound of the number of allocations at cost $\hat{c}_{(h-1)}$ in the positions corresponding to the null blocks. In the same way, the 2nd to the 4-th sums in (39) provide an upper bound on the number of these allocations.

\section{Computational Results}

The formulations given to the CSA-OMHLP with the corresponding strengthening and preproccesing phases, described in this paper, were implemented in the commercial solver XPRESS IVE 1.23.02.64 running on a Intel(R) Core(TM) i5-3450 CPU @3.10GHz6GB RAM.

The cut generation option of XPRESS was disabled in order to compare the relative performance of the formulations cleanly.

For this purpose we use the AP data set publicly available at http://www.cmis.csiro.au/or /hubLocation (see [19]). As in previous papers on the field related to the uncapacitated version of this problem, we tested the formulations on a testbed of five instances for each combination of costs matrices varying: (i) $n$ in $\{15,20,25,28,30\}$ (ii) three different values of $p$ depending on the case and (iii) $\mu=0.7, \delta=0.9 \mu$ and six different $\lambda$-vectors. These $\lambda$-vectors are the wellknown Median $\lambda=(1, \ldots, 1)$, Anti- $\left(k_{1}+k_{2}\right)$-trimmed-mean $\lambda=\left(1, k_{1}, 1,0, \ldots, 0,1, k_{2} ., 1\right)$, $\left(k_{1}+k_{2}\right)$-Trimmed-mean $\lambda=\left(0, k_{1} ., 0,1, \ldots, 1,0, k_{2} ., 0\right)$, with $k_{1}=k_{2}=\lceil 0.2 n\rceil$, Center $\lambda=$ $(0, \ldots, 0,1)$, and $k$-Centrum $\lambda=(0, \ldots, 0,1, . k ., 1)$ with $k=\lceil 0.2 n\rceil$. As well as a $\{0,1\}$-blocks $\lambda$-vector (three alternate $\{0-1\}$-blocks of lambda weights, i.e. $\lambda=(0, \ldots, 0,1, \ldots, 1,0, \ldots, 0$, 


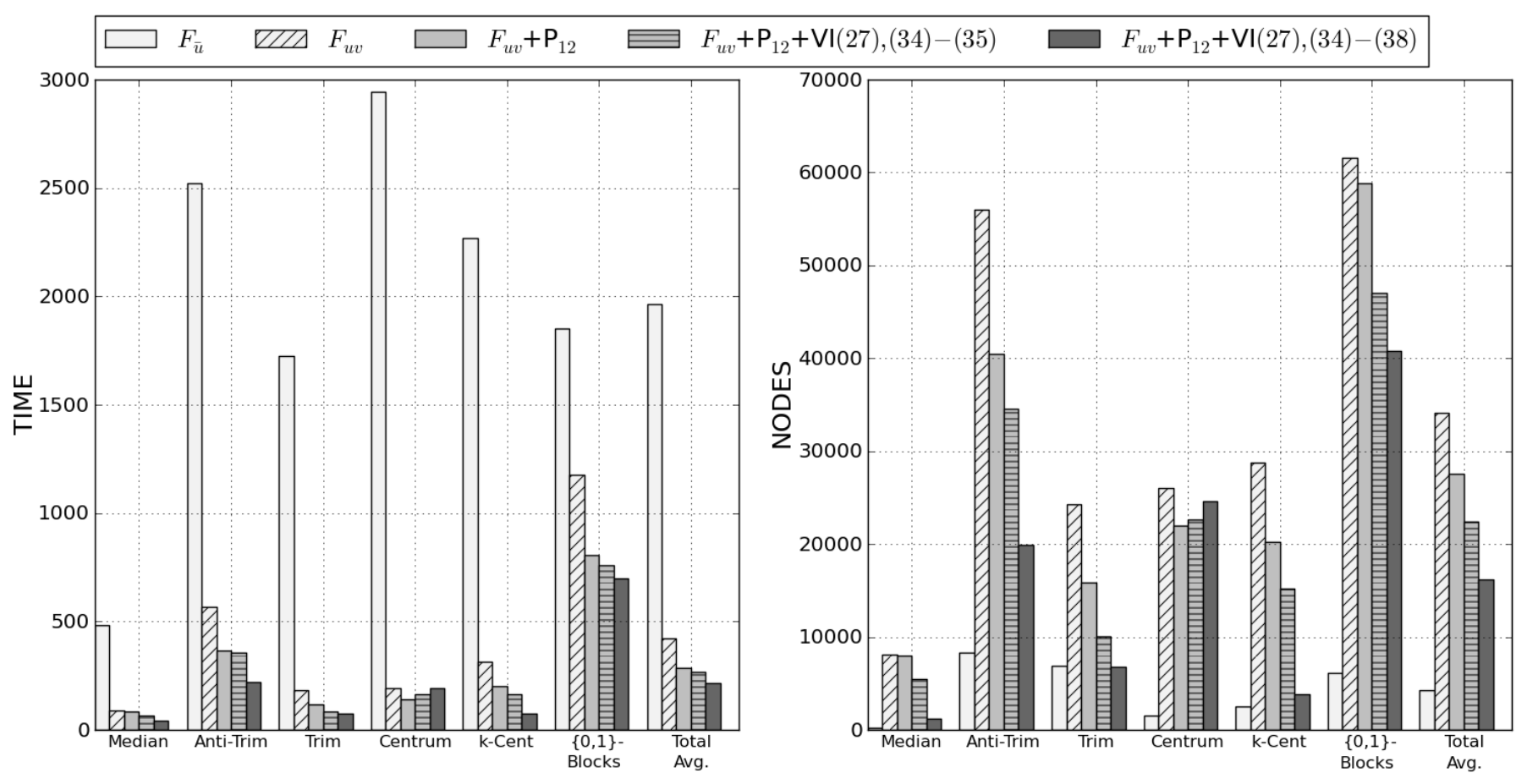

Figure 3: Summary of computational results carried out in the paper.

$1, \ldots, 1,0, \ldots, 0,1, \ldots, 1))$. Therefore, for the each combination of $n, p$ and $\lambda$ we have tested five instances. This is, a total number of 450 problems have been used to test the performance of the proposed models.

The capacities were randomly generated in $\left[\min _{i} W_{i},(1 / 2) \sum_{i \in A} W_{i}\right]$. This generation procedure does not ensure in all cases feasible instances, as capacity constraints can be very tight for problems with a low number of hubs $(n=3,5)$. Overall, in our experiments we got, initially 10 infeasible instances out of 75 (13.3\%). These instances were replaced by new feasible ones (generated with the same capacity structure). Hence, the reader may observe that the generation procedure gives tight capacity constraints.

First of all, and for the sake of readability, we present in Figure 3 a summary of our computational results. A full description of those results is also included in Tables [1,6.

Figure 3 shows average results for each one of the considered problem types (different $\lambda$-vectors). The left chart refers to average CPU times and the right chart to the number of 
explored nodes of the $\mathrm{B} \& \mathrm{~B}$ tree. Both charts contain the same number of blocks standing for the different types of $\lambda$-vectors plus and additional block (Total Avg.) for the consolidated average of all $\lambda$-vectors. Each block compares the behavior of the different formulations $\left(F_{\bar{u}}, F_{u v}\right)$ and their strengthening (variable fixing and valid inequalities). The heading explains the meaning of bars within each block: $F_{\bar{u}}$ and $F_{u v}$ stand for the corresponding formulations; $F_{u v}+\mathrm{P}_{12}$ when the two preprocesses $P_{1}(h)$ and $P_{2}(h)$ are applied; and $F_{u v}+\mathrm{P}_{12}+$ VI when the Valid Inequalities are added as well, denoted with their corresponding references.

Analyzing this figure we observe the improvement obtained with $F_{u v}$ and its strengthening as compared with $F_{\bar{u}}$ or even $F_{u v}$ alone. Actually, the overall reduction in running time with respect to the initial formulation is above $89 \%$.

Next, focusing in the best model, namely $F_{u v}+P_{12}+\mathrm{VI}(\underline{28})$, (35)-(39), we observe that the most time consuming problem is the one with $\lambda$-vector given by $\{0,1\}$-blocks with a significant difference with respect to the remaining $\lambda$-vectors. The second most time consuming problem corresponds with the Antitrimmean. Similar conclusions, regarding the number of nodes, are obtained looking at the right chart of Figure 3. It is worth mentioning that although the formulation $F_{\bar{u}}$ provides the worst computational times, it is the one that reports the lowest number of nodes in the $\mathrm{B} \& \mathrm{~B}$ tree. This fact is explained because $F_{\bar{u}}$ has a larger number of variables than $F_{u v}$ which results in more difficult LP relaxations in each node of the B\&B tree.

In spite of that Figure 3 shows the general overview of our computational results, we also include in the following a more detailed analysis based on Tables 1-6.

Tables 1 and 2 report the results of the formulations and different preprocessing phases developed in this paper for the CSA-OMHLP. The first column of these tables describes the different $\lambda$-vectors in the study, the second and third columns report the size of the instances and the number of hubs to be located, respectively. The following three columns correspond to some of the computational results obtained by solving the CSA-OMHLP with $F_{\bar{u}}$ formulation. The next three columns correspond to Formulation $F_{u v}$, and the rest of the columns to the latter formulation plus the two preprocessing procedures, i.e $F_{u v}+\mathrm{P}_{1}+\mathrm{P}_{2}$. Columns $R G A P$, Nodes and Time stand for the averages of: the gap in the root node, number of nodes in the 
$\mathrm{B} \& \mathrm{~B}$ tree and the CPU time in seconds; the time was limited to two hours of CPU. To obtain a general idea of the comparisons among these averaged values, for the results in the column Time and for different formulations and/or valid inequalities applied, we have accounted the value 7200 seconds for those instances that exceed the time limit. A superindex in their corresponding averaged time value states the number of instances exceeding the CPU time limit; in the same way, the values used to computed the average of the column Nodes have been the number of nodes of the B\&B tree when the CPU time limit was reached.

The column Fixed gives the percentage of variables that have been fixed after the Preprocessing Phases 1 and 2. Column Cuts provides the number of the lower and upper bounds over the variables $v$ added to the model, after running the corresponding preprocessing phases. Finally, T. prep. reports the CPU time in seconds of the corresponding preprocessing phases and column T. total reports the overall CPU time in seconds to solve the problem including the corresponding preprocessing phase. The rows Average provide the averaged results among all the tested instances for each problem and TOTAL with respect to the overall set of instances.

Tables 1 and 2 show, as a general trend, that the CPU time increases similarly, for all choices of the $\lambda$-vector, with the size of the instances. We can see that 69 instances required more than two hours to be solved using Formulation $F_{\bar{u}}$, however the remaining two analyzed ways to solve this problem were able to solve all the instances within the CPU time limit, except in a few cases for the Antitrimmean and $\{0,1\}$-blocks problem types. Regarding the running times for the different types of problems, we can see that Anti-TrimMean and Blocks have been the problems that need more time to be solved. In any case, we observed that there is a considerable reduction of the running times from the original formulation to the improved formulation, and after applying the preprocessing phases. From tables 11 and 2 , one can remark that, Formulation $F_{u v}$ with Preprocessing Phases 1 and 2 provides better results, with a reduction of around a $86 \%$ of the time with respect to Formulation $F_{\bar{u}}$ (taking into account that the latter formulation was not able to solve all the studied instances before the time limit was exceeded). Moreover, the reduction of the running times with respect to Formulation $F_{u v}$ (without preprocessing phases) is around $32 \%$. 
As for the comparison between RGAP's, we observe that the average gap of the linear relaxation after preprocessing reduces around $32 \%$ from the original formulation (Formulation $\left.F_{\bar{u}}\right)$ to Formulation $F_{u v}$ with Preprocessing Phases 1 and 2 . In any case, it is also worth noting that the gap from the original formulation to the improved formulation (without any preprocessing phase) is reduced around 9\%, what implies that even though this improved formulation uses much less number of variables, it provides a better RGAP.

Tables 3 and 4 present several improvements to Formulation $F_{u v}$ with Preprocessing Phases 1 and 2. In particular, the second, third and fourth blocks of columns summarize the results when the combination of valid inequalities (28)-(30); (28), (33)-(34) and (28), (35)-(36) are added to this formulation, respectively. In general, we can see that the latter combination provides the best results. In particular, there is an improvement of $7 \%$ of the running times with respect to Formulation $F_{u v}$ with Preprocessing Phases 1 and 2. Moreover, with respect to the number of nodes the improvement is around 15\%, but the RGAP is similar for all the analyzed reinforcements.

Since the best behavior observed was obtained with reinforcement given by (28), (35)-(36), in the rest of our tests we have used this configuration to make further strengthening. Tables 5 and 6 present several improvements to the Formulation $F_{u v}$ with Preprocessing Phases 1 and 2 and valid inequalities (28), (35)-(36). In particular, the second, third and fourth blocks of columns report the results after including the family of valid inequalities (37), (37)-(38) and (37)-(39), respectively. These tables show that the best results are obtained when all valid inequalities, (37)-(39), are added to the current configuration; with an improvement of $18 \%$ in the running time, $28 \%$ in the number of nodes and $12 \%$ in the RGAP.

The overall conclusion of our experiment is that in order to solve CSA-OMHLP the best combination of formulation and strengthening is to use $F_{u v}+P_{12}+(\underline{28})+(\underline{35})+(\underline{36})+(37)+(39)$. This configuration allows to solve medium size instances within 10 minutes of CPU time.

\section{Concluding remarks}

This paper can be considered as an initial attempt to address the capacitated single-allocation ordered hub location problem. The formulations, strengthening and preprocessing phases de- 
veloped in this paper provide a promising approach to solve the above mentioned problem although so far only medium size problems are reasonably well-solved. Thus, this work opens interesting possibilities to study-and-develop ad-hoc solution procedures that allow us to consider larger size instances of this problem. Moreover, it also points out the possibility of developing heuristics approaches that will give good solutions in competitive running times. All in all, this paper shows the usefulness of using covering formulations and their corresponding strengthening for solving capacitated versions of ordered hub location problems.

\section{Acknowledgement}

This research has been partially supported by Spanish Ministry of Education and Science/FEDER grants numbers MTM2010-19576-C02-(01-02), MTM2013-46962-C02-(01-02), Junta de Andalucía grant number FQM 05849 and Fundación Séneca, grant number 08716/PI/08.

\section{References}

[1] S. Alumur and B.Y. Kara. Network hub location problems: the state of the art. European Journal of Operational Research, 190(1):1-21, 2008.

[2] T. Aykin Lagrangian relaxation based approaches to capacitated hub-and-spoke network design problem. European Journal of Operational Research, 79(3):501-523, 1994.

[3] N. Boland, P. Domínguez-Marín, S. Nickel, and J. Puerto. Exact procedures for solving the discrete ordered median problem. Computers and Operations Research, 33:3270$3300,2006$.

[4] R. Bollapragada, Y. Li, and U.S. Rao. Budget-constrained, capacitated hub location to maximize expected demand coverage in fixed-wireless telecommunication networks. INFORMS Journal on Computing, 18(4):422-432, 2006.

[5] J.F. Campbell. Integer programming formulations of discrete hub location problems. European Journal of Operational Research, 72:387-405, 1994. 
[6] J.F. Campbell. Hub location and the p-hub median problem. Operations Research, 44(6):923-935, 1996.

[7] J.F. Campbell, A. Ernst, and M. Krishnamoorthy. Hub location problems. Facility location, 373-407, Springer, Berlin, 2002.

[8] A.M. Campbell, T.J. Lowe, and L. Zhang. The $p$-hub center allocation problem. European Journal of Operational Research, 176(2):819-835, 2007.

[9] J.F. Campbell and M.E. O'Kelly. Twenty five years of hub location research. Transportation Science, 46 (2):153-169, 2012.

[10] L. Cánovas, S. García, and A. Marín. Solving the uncapacitated multiple allocation hub location problem by means of a dual-ascent technique. European Journal of Operational Research, 179(3):990-1007, 2007.

[11] I. Contreras, J.A. Díaz, and E. Fernández. Lagrangean relaxation for the capacitated hub location problem with single assignment. OR Spectrum, 31(3):483-505, 2009.

[12] I. Contreras, J.F. Cordeau, G. Laporte. Benders decomposition for large-scale uncapacitated hub location. Operations Research, 59, 1477-1490, 2011.

[13] I. Correia, S. Nickel, F. Saldanha-da-Gama. The capacitated single-allocation hub location problem revisited: A note on a classical formulation. European Journal of Operational Research, 207(1):92-96, 2010.

[14] I. Correia, S. Nickel, and F. Saldanha-da-Gama. Single-assignment hub location problems with multiple capacity levels. Transportation Research Part B, 44:1047-1066, 2010.

[15] J. Ebery, M. Krishnamoorthy, A. Ernst, N. Boland. The capacitated multiple allocation hub location problem: Formulations and algorithms. European Journal of Operational Research, 120(3):614-631, 2000.

[16] N. Boland, M. Krishnamoorthy, A. Ernst, J. Ebery. Preprocessing and cutting for multiple allocation hub location problems. European Journal of Operational Research, 155(3):638-653, 2004. 
[17] R.S. De Camargo, G.J. Miranda, R.P.M. Ferreira, H.P. Luna. Multiple allocation huband-spoke network design under hub congestion. Computers and Operations Research, 36(12):3097-3106, 2009.

[18] S. Elhedhli, F. Hu. Hub-and-spoke network design with congestion. Computers and Operations Research, 32(6):1615-1632, 2004.

[19] A.T. Ernst an M. Krishnamoorthy. Efficient algorithms for the uncapacitated single allocation p-hub median problem. Location Science, 4(3):139-154, 1996.

[20] A.T. Ernst and M. Krishnamoorthy. Solution algorithms for the capacitated single allocation hub location problem. Annals of Operations Research, 86:141-159, 1999.

[21] M.C. Fonseca, A. García-Sánchez, M. Ortega-Mier, and F. Saldanha-da-Gama. A stochastic bi-objective location model for strategic reverse logistics. TOP, 18(1):158$184,2010$.

[22] J. Kalcsics, S. Nickel, J. Puerto, and A.M. Rodríguez-Chía. Distribution systems design with role dependent objectives. European Journal of Operational Research, 202:491-501, 2010.

[23] J. Kalcsics, S. Nickel, J. Puerto, and A.M. Rodríguez-Chía. The ordered capacitated facility location problem. TOP, 18(1):203-222, 2010.

[24] B.Y. Kara and B.C. Tansel. On the single-assignment p-hub center problem. European Journal of Operational Research, 125(3):648-655, 2000.

[25] B.Y. Kara, and B.C. Tansel. The single-assignment hub covering problem: Models and linearizations. Journal of the Operational Research Society, 54:59-64, 2003.

[26] J. Kratica and Z. Stanimirovic. Solving the uncapacitated multiple allocation $p$-hub center problem by genetic algorithm. Asia-Pacific Journal of Operational Research, 23(4):425-437, 2006.

[27] M. Labbé, H. Yaman, and E. Gourdin. A branch and cut algorithm for hub location problems with single assignment. Mathematical Programming, 102(2):371-405, 2005. 
[28] V. Marianov, D. Serra. Location models for airline hubs behaving as M/D/c queues. Computers and Operations Research, 30(7):983-1003, 2003.

[29] A. Marín. Formulating and solving splittable capacitated multiple allocation hub location problems. Computers and Operations Research, 32(12):3093-3109, 2005.

[30] A. Marín. Uncapacitated Euclidean hub location: strengthened formulation, new facets and a relax-and-cut algorithm. Journal of Global Optimization, 33(3):393-422, 2005.

[31] A. Marín, L. Cánovas, and M. Landete. New formulations for the uncapacitated multiple allocation hub location problem. European Journal of Operational Research, 172(1):274$292,2006$.

[32] A. Marín, S. Nickel, J. Puerto, and S. Velten. A flexible model and efficient solution strategies for discrete location problems. Discrete Applied Mathematics, 157(5):1128$1145,2009$.

[33] A. Marín, S. Nickel, and S. Velten. An extended covering model for flexible discrete and equity location problems. Mathematical Methods of Operations Research, 71(1):125-163, 2010.

[34] T. Meyer, A.T. Ernst, and M. Krishnamoorthy. A 2-phase algorithm for solving the single allocation p-hub center problem. Computers and Operations Research, 36(12):31433151, 2009.

[35] S. Nickel and J. Puerto. Location Theory - A Unified Approach. Springer, 2005.

[36] J. Puerto, A.B. Ramos, and A.M. Rodríguez-Chía. Single-Allocation Ordered Median Hub Location Problems. Computers and Operations Research, 38:559-570, 2011.

[37] J. Puerto, A.B. Ramos, and A.M. Rodríguez-Chía. A specialized Branch \& Bound \& Cut for Single-Allocation Ordered Median Hub Location Problems. Discrete Applied Mathematics, 161:2624-2646, 2013.

[38] A.B. Ramos. Hub Location Problems with Ordered Median (In Spanish). Ph.D. Thesis. Facultad de Matemáticas, Universidad de Sevilla, 2012. 
[39] D. Skorin-Kapov, J. Skorin-Kapov, and M. O'Kelly. Tight linear programming relaxations of uncapacitated $p$-hub median problems. European Journal of Operational Research, 94:582-593, 1996.

[40] P.Z. Tan and B.Y. Kara. A hub covering model for cargo delivery systems. Networks, 49(1):28-39, 2007.

[41] B. Wagner. Model formulations for hub covering problems. Journal of the Operational Research Society, 59(7):932-938, 2008. 
Table 1: Computational results for Formulations $F_{\bar{u}}$ vs $F_{u v}$ with preprocessing.

\begin{tabular}{|c|c|c|c|c|c|c|c|c|c|c|c|c|c|c|}
\hline & \multirow{2}{*}{$\mathrm{n}$} & \multirow{2}{*}{$\mathrm{p}$} & \multicolumn{3}{|c|}{ Formulation $F_{\bar{u}}$} & \multicolumn{3}{|c|}{ Formulation $F_{u v}$} & \multicolumn{6}{|c|}{ Formulation $F_{u v}+\mathbf{P}_{1}+\mathbf{P}_{2}$} \\
\hline & & & RGAP & Nodes & Time & RGAP & Nodes & Time & RGAP & Nodes & $\mathrm{T}$ total & T. prep & Fixed & Cuts \\
\hline \multirow{16}{*}{$\begin{array}{l}\text { 壱 } \\
\text { 畺 }\end{array}$} & 15 & 3 & 17,56 & 17 & 7,9 & 17,56 & 240 & 1,8 & 15,16 & 195 & 1,4 & 1,4 & 2,4 & 240 \\
\hline & 15 & 5 & 7,60 & 3 & 3,8 & 7,60 & 230 & 0,8 & 6,78 & 177 & 0,7 & 1,2 & 1,4 & 180 \\
\hline & 15 & 8 & 8,49 & 1 & 1,0 & 8,49 & 259 & 0,6 & 4,39 & 185 & 0,4 & 1,0 & 0,8 & 133 \\
\hline & 20 & 3 & 19,54 & 75 & 69,5 & 19,54 & 505 & 6,3 & 16,31 & 472 & 5,8 & 3,1 & 3,9 & 405 \\
\hline & 20 & 8 & 6,05 & 1 & 3,9 & 6,05 & 1024 & 4,2 & 5,49 & 1122 & 3,8 & 2,2 & 1,9 & 215 \\
\hline & 20 & 10 & 2,88 & 1 & 3,1 & 2,88 & 591 & 1,9 & 2,59 & 560 & 1,6 & 1,7 & 1,8 & 181 \\
\hline & 25 & 3 & 19,00 & 1.051 & 509,5 & 19,00 & 478 & 21,2 & 15,21 & 408 & 18,5 & 8,9 & 7,2 & 800 \\
\hline & 25 & 8 & 6,77 & 1 & 12,7 & 6,77 & 4239 & 30,9 & 5,95 & 6177 & 33,6 & 5,2 & 4,1 & 459 \\
\hline & 25 & 10 & 13,10 & 15 & 48,6 & 13,10 & 6656 & 33,4 & 6,31 & 4686 & 26,0 & 5,2 & 3,4 & 435 \\
\hline & 28 & 3 & 21,86 & 270 & 322,7 & 21,86 & 2052 & 66,8 & 16,01 & 2246 & 76,6 & 13,2 & 10,5 & 960 \\
\hline & 28 & 8 & 9,21 & 143 & 415,6 & 9,21 & 16820 & 165,5 & 8,15 & 10560 & 97,4 & 7,7 & 6,5 & 597 \\
\hline & 28 & 10 & 7,36 & 129 & 321,3 & 7,36 & 27440 & 193,1 & 6,78 & 32680 & 243,1 & 6,7 & 3,8 & 396 \\
\hline & 30 & 3 & 32,36 & 1.416 & $4007,0^{(1)}$ & 32,32 & 3701 & 167,7 & 20,92 & 2564 & 127,8 & 18,8 & 11,0 & 1128 \\
\hline & 30 & 8 & 18,69 & 360 & 1346,0 & 18,69 & 39430 & 455,5 & 10,86 & 36790 & 437,3 & 12,8 & 6,2 & 817 \\
\hline & 30 & 10 & 9,18 & 22 & 186,3 & 9,18 & 18420 & 176,8 & 6,98 & 20130 & 207,6 & 11,4 & 5,4 & 685 \\
\hline & \multicolumn{2}{|c|}{ Average } & 13,30 & 234 & 483,9 & 13,30 & 8139 & 88,4 & 9,90 & 7930 & 85,4 & 6,7 & 4,7 & 509 \\
\hline \multirow{16}{*}{ 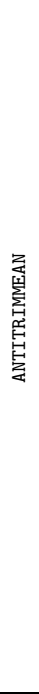 } & 15 & 3 & 23,75 & 150 & 33,6 & 23,75 & 614 & 2,8 & 17,62 & 645 & 2,0 & 1,4 & 6,1 & 164 \\
\hline & 15 & 5 & 13,93 & 99 & 12,9 & 13,93 & 1039 & 2,2 & 8,38 & 803 & 1,4 & 1,1 & 4,3 & 104 \\
\hline & 15 & 8 & 13,20 & 29 & 9,4 & 13,20 & 618 & 1,1 & 6,82 & 724 & 0,9 & 1,0 & 2,6 & 91 \\
\hline & 20 & 3 & 23,50 & 2448 & 272,0 & 23,50 & 2032 & 16,8 & 17,44 & 1150 & 9,2 & 3,1 & 9,8 & 273 \\
\hline & 20 & 8 & 9,45 & 379 & 79,9 & 9,45 & 4637 & 11,9 & 7,66 & 3009 & 7,8 & 2,2 & 5,2 & 126 \\
\hline & 20 & 10 & 4,66 & 73 & 36,4 & 4,66 & 3150 & 6,9 & 4,28 & 3394 & 6,4 & 1,7 & 4,4 & 123 \\
\hline & 25 & 3 & 24,64 & 12130 & 2037,0 & 24,64 & 1984 & 53,8 & 17,29 & 1218 & 29,0 & 8,9 & 18,9 & 540 \\
\hline & 25 & 8 & 10,32 & 590 & 344,4 & 10,32 & 6411 & 40,2 & 7,91 & 6841 & 33,3 & 5,2 & 10,4 & 324 \\
\hline & 25 & 10 & 17,25 & 13650 & 1217,0 & 17,25 & 27180 & 115,1 & 9,81 & 25390 & 102,1 & 5,2 & 8,6 & 330 \\
\hline & 28 & 3 & 25,94 & 16460 & $5111,0^{(1)}$ & 25,94 & 26600 & 957,1 & 17,36 & 7432 & 168,4 & 13,2 & 22,1 & 717 \\
\hline & 28 & 8 & 13,58 & 12390 & $5688,0^{(3)}$ & 13,52 & 42860 & 381,4 & 10,67 & 34800 & 271,1 & 7,8 & 16,8 & 301 \\
\hline & 28 & 10 & 10,95 & 13830 & $3524,0^{(2)}$ & 10,92 & 262500 & 1595,0 & 9,14 & 131900 & 832,1 & 6,7 & 9,7 & 242 \\
\hline & 30 & 3 & 36,85 & 11110 & $6584,0^{(4)}$ & 35,55 & 15670 & 591,0 & 21,74 & 7220 & 271,6 & 18,8 & 27,2 & 770 \\
\hline & 30 & 8 & 23,80 & 19960 & $7200,0^{(5)}$ & 23,72 & 299700 & 3402,0 & 14,48 & 273600 & 2896,0 & 12,8 & 15,8 & 598 \\
\hline & 30 & 10 & 13,53 & 21200 & $5707,0^{(3)}$ & 13,50 & 145000 & 1310,0 & 10,56 & 109700 & 863,2 & 11,4 & 15,4 & 464 \\
\hline & \multicolumn{2}{|c|}{ Average } & 17,70 & 8300 & 2523,8 & 17,60 & 56000 & 565,8 & 12,10 & 40522 & 366,3 & 6,7 & 11,8 & 345 \\
\hline \multirow{16}{*}{$\begin{array}{l}\text { 离 } \\
\text { 离 } \\
\text { 䍃 }\end{array}$} & 15 & 3 & 19,46 & 177 & 22,9 & 17,43 & 1066 & 2,1 & 16,44 & 729 & 1,4 & 1,4 & 0,8 & 76 \\
\hline & 15 & 5 & 11,26 & 38 & 13,6 & 8,65 & 1209 & 1,1 & 8,58 & 646 & 0,7 & 1,2 & 0,8 & 67 \\
\hline & 15 & 8 & 8,72 & 4 & 3,6 & 7,88 & 510 & 0,4 & 7,88 & 322 & 0,3 & 1,0 & 0,3 & 20 \\
\hline & 20 & 3 & 20,77 & 5452 & 335,9 & 19,38 & 1047 & 7,0 & 17,65 & 1022 & 6,2 & 3,1 & 1,9 & 132 \\
\hline & 20 & 8 & 12,10 & 1107 & 96,7 & 9,48 & 6847 & 13,4 & 9,08 & 4512 & 7,9 & 2,2 & 1,2 & 88 \\
\hline & 20 & 10 & 8,88 & 63 & 36,7 & 6,17 & 2309 & 4,4 & 5,97 & 1620 & 2,7 & 1,7 & 0,9 & 58 \\
\hline & 25 & 3 & 19,74 & 18180 & 2157,0 & 18,78 & 2039 & 31,4 & 16,87 & 2081 & 25,1 & 8,9 & 3,2 & 260 \\
\hline & 25 & 8 & 10,55 & 5365 & 804,0 & 8,94 & 61330 & 211,7 & 8,63 & 21460 & 74,2 & 5,2 & 2,2 & 140 \\
\hline & 25 & 10 & 13,58 & 1186 & 225,4 & 12,34 & 2642 & 9,6 & 12,28 & 1760 & 6,5 & 5,2 & 1,9 & 117 \\
\hline & 28 & 3 & 21,50 & 18050 & $4959,0^{(1)}$ & 20,54 & 8611 & 149,2 & 17,49 & 3800 & 69,6 & 13,3 & 3,9 & 243 \\
\hline & 28 & 8 & 12,23 & 12740 & 2304,0 & 10,74 & 47430 & 309,6 & 10,24 & 20290 & 130,9 & 7,7 & 3,8 & 234 \\
\hline & 28 & 10 & 11,27 & 6731 & 1467,0 & 9,25 & 35750 & 178,7 & 9,171 & 14770 & 69,3 & 6,7 & 2,8 & 174 \\
\hline & 30 & 3 & 26,67 & 9820 & $6506,0^{(4)}$ & 25,15 & 9904 & 294,1 & 22,08 & 3302 & 124,4 & 18,8 & 5,6 & 358 \\
\hline & 30 & 8 & 17,04 & 10390 & $3766,0^{(1)}$ & 15,44 & 90810 & 868,1 & 15,09 & 54920 & 483,1 & 12,8 & 3,6 & 243 \\
\hline & 30 & 10 & 11,37 & 14330 & 3181,0 & 9,44 & 92100 & 690,0 & 9,40 & 107100 & 757,0 & 11,4 & 3,2 & 227 \\
\hline & \multicolumn{2}{|c|}{ Average } & 15,00 & 6909 & 1725,3 & 13,30 & 24240 & 184,7 & 12,50 & 15889 & 117,3 & 6,7 & 2,4 & 163 \\
\hline
\end{tabular}


Table 2: Computational results for Formulations $F_{\bar{u}}$ vs $F_{u v}$ with preprocessing(II).

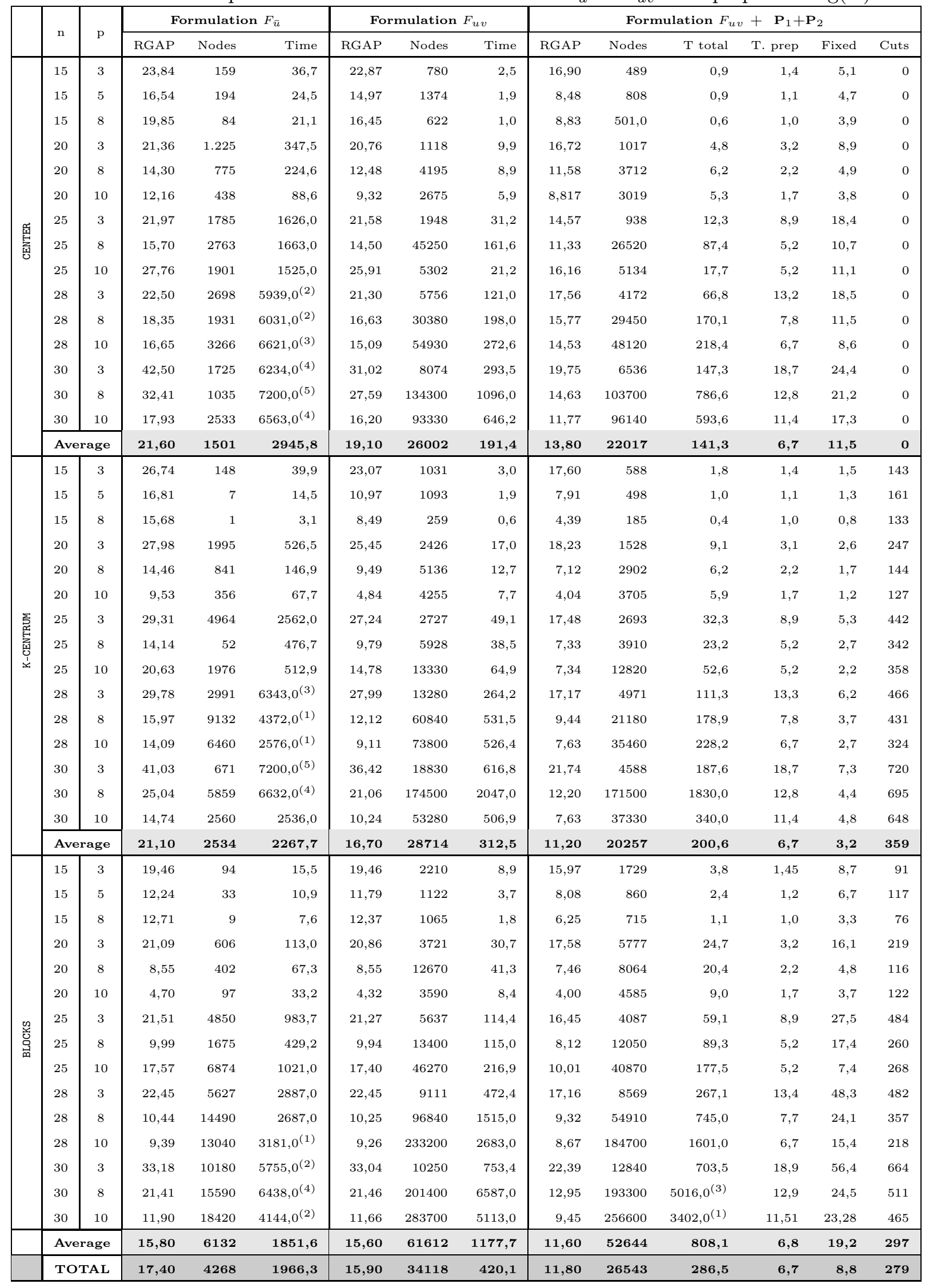


Table 3: Computational results for Formulation $F_{u v}$ with Valid Inequalities (I)

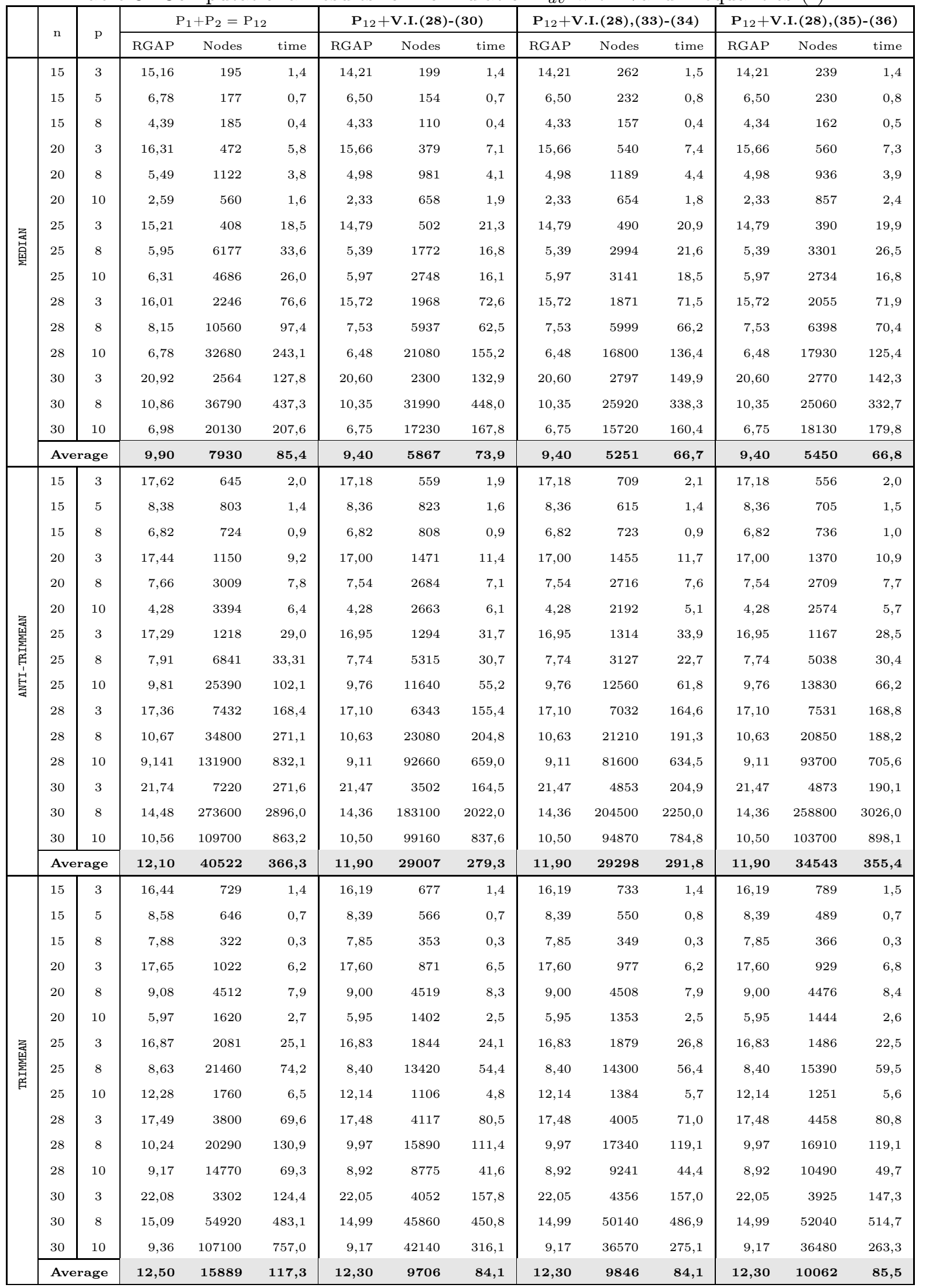


Table 4: Computational results for Formulation $F_{u v}$ with Valid Inequalities (I)

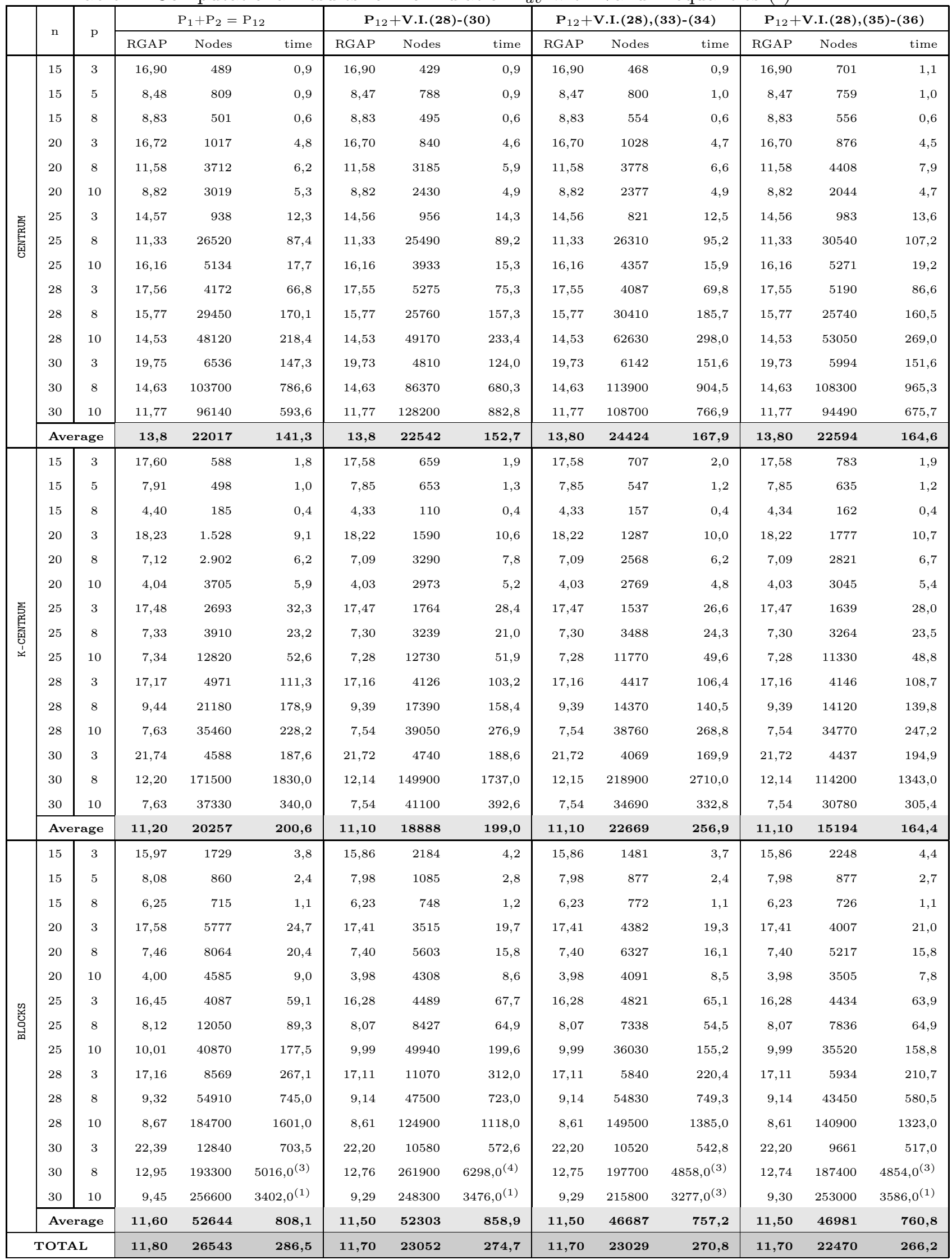


Table 5: Computational results for Formulation $F_{u v}$ with Valid Inequalities (II)

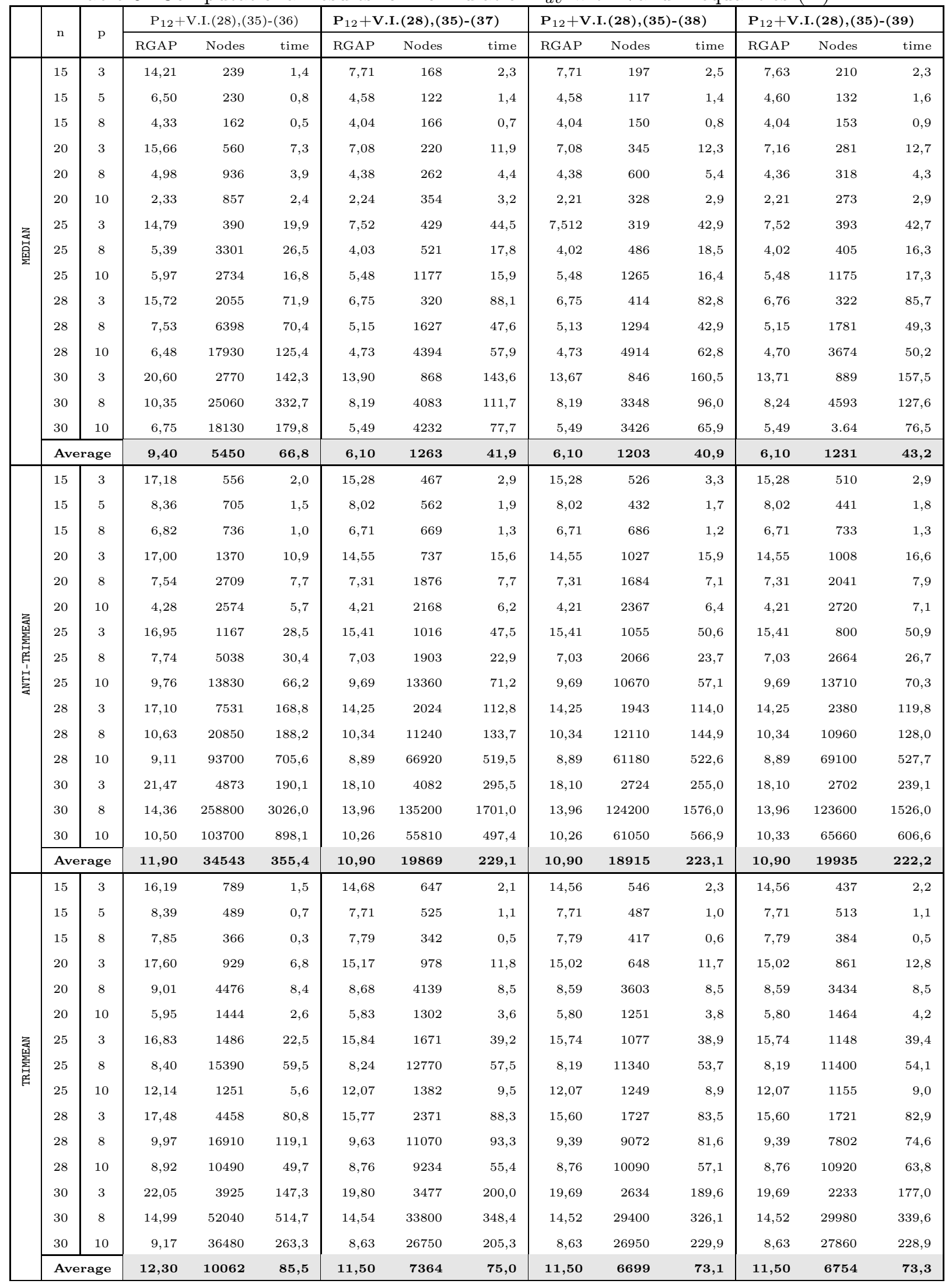


Table 6: Computational results for Formulation $F_{u v}$ with Valid Inequalities (II) bis

\begin{tabular}{|c|c|c|c|c|c|c|c|c|c|c|c|c|c|c|}
\hline & \multirow{2}{*}{$\mathrm{n}$} & \multirow{2}{*}{$\mathrm{p}$} & \multicolumn{3}{|c|}{$\mathrm{P}_{12}+$ V.I. 28, , 35)- 36} & \multicolumn{3}{|c|}{$\mathbf{P}_{12}+$ V.I. 28, , $35-(37)$} & \multicolumn{3}{|c|}{ P $_{12}+$ V.I. $(28),(35)-(38)$} & \multicolumn{3}{|c|}{$\mathbf{P}_{12}+$ V.I. $28,(35)-(39)$} \\
\hline & & & RGAP & Nodes & time & RGAP & Nodes & time & RGAP & Nodes & time & RGAP & Nodes & time \\
\hline \multirow{16}{*}{$\begin{array}{l}\text { 总 } \\
\text { 焉 }\end{array}$} & 15 & 3 & 16,90 & 701 & 1,1 & 16,32 & 574 & 1,6 & 16,32 & 576 & 1,6 & 16,32 & 513 & 1,6 \\
\hline & 15 & 5 & 8,47 & 759 & 1,0 & 8,45 & 769 & 1,3 & 8,45 & 850 & 1,4 & 8,45 & 850 & 1,5 \\
\hline & 15 & 8 & 8,83 & 556 & 0,6 & 8,81 & 577 & 0,8 & 8,78 & 582 & 0,9 & 8,78 & 437 & 0,9 \\
\hline & 20 & 3 & 16,70 & 876 & 4,5 & 15,94 & 579 & 7,1 & 15,94 & 649 & 7,0 & 15,94 & 705 & 6,9 \\
\hline & 20 & 8 & 11,58 & 4408 & 7,9 & 11,57 & 4061 & 7,9 & 11,56 & 3902 & 8,3 & 11,56 & 3725 & 7,8 \\
\hline & 20 & 10 & 8,82 & 2044 & 4,7 & 8,82 & 2044 & 5,4 & 8,82 & 2739 & 6,2 & 8,82 & 2389 & 5,6 \\
\hline & 25 & 3 & 14,56 & 983 & 13,6 & 14,27 & 689 & 19,0 & 14,27 & 669 & 19,8 & 14,27 & 750 & 19,9 \\
\hline & 25 & 8 & 11,33 & 30540 & 107,2 & 11,29 & 29000 & 103,6 & 11,29 & 25290 & 96,5 & 11,29 & 28560 & 104,6 \\
\hline & 25 & 10 & 16,16 & 5271 & 19,2 & 16,15 & 4339 & 18,4 & 16,15 & 4335 & 18,6 & 16,15 & 5226 & 21,4 \\
\hline & 28 & 3 & 17,55 & 5190 & 86,6 & 16,87 & 2660 & 74,6 & 16,87 & 2788 & 79,9 & 16,87 & 2997 & 83,0 \\
\hline & 28 & 8 & 15,77 & 25740 & 160,5 & 15,75 & 26910 & 175,6 & 15,75 & 25320 & 179,3 & 15,75 & 26240 & 173,8 \\
\hline & 28 & 10 & 14,53 & 53050 & 269,0 & 14,52 & 57390 & 296,1 & 14,52 & 58690 & 314,7 & 14,52 & 50760 & 265,1 \\
\hline & 30 & 3 & 19,73 & 5994 & 151,6 & 18,36 & 4419 & 183,1 & 18,36 & 3236 & 148,0 & 18,36 & 4846 & 190,2 \\
\hline & 30 & 8 & 14,63 & 108300 & 965,3 & 14,59 & 119500 & 1068,0 & 14,59 & 110300 & 1094,0 & 14,59 & 133400 & 1228,0 \\
\hline & 30 & 10 & 11,77 & 94490 & 675,7 & 11,77 & 94490 & 678,4 & 11,77 & 91310 & 700,3 & 11,77 & 107000 & 806,9 \\
\hline & \multicolumn{2}{|c|}{ Average } & 13,80 & 22594 & 164,6 & 13,60 & 23200 & 176,1 & 13,60 & 22082 & 178,4 & 13,60 & 24560 & 194,5 \\
\hline \multirow{16}{*}{ 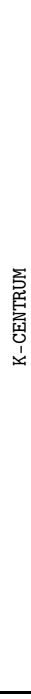 } & 15 & 3 & 17,58 & 783 & 1,9 & 14,72 & 678 & 3,3 & 14,49 & 502 & 3,2 & 14,49 & 594 & 3,4 \\
\hline & 15 & 5 & 7,85 & 6345 & 1,2 & 6,80 & 437 & 1,8 & 6,58 & 387 & 1,8 & 6,58 & 358 & 1,9 \\
\hline & 15 & 8 & 4,34 & 162 & 0,4 & 4,04 & 166 & 0,7 & 4,04 & 150 & 0,8 & 4,04 & 153 & 0,8 \\
\hline & 20 & 3 & 18,22 & 1777 & 10,7 & 15,52 & 959 & 13,3 & 15,25 & 847 & 14,1 & 15,25 & 904 & 13,8 \\
\hline & 20 & 8 & 7,09 & 2821 & 6,7 & 6,77 & 1272 & 6,1 & 6,62 & 1260 & 6,7 & 6,62 & 1001 & 6,4 \\
\hline & 20 & 10 & 4,03 & 3045 & 5,4 & 3,84 & 2437 & 6,0 & 3,74 & 1879 & 5,6 & 3,74 & 1950 & 5,5 \\
\hline & 25 & 3 & 17,47 & 1639 & 28,0 & 16,17 & 1404 & 46,7 & 16,11 & 1035 & 45,3 & 16,11 & 1377 & 50,7 \\
\hline & 25 & 8 & 7,29 & 3264 & 23,5 & 6,46 & 1895 & 23,2 & 6,30 & 1777 & 23,8 & 6,30 & 1571 & 23,1 \\
\hline & 25 & 10 & 7,28 & 11330 & 48,8 & 7,09 & 6548 & 38,5 & 6,99 & 5529 & 36,0 & 6,99 & 5793 & 37,2 \\
\hline & 28 & 3 & 17,16 & 4146 & 108,7 & 14,52 & 1557 & 83,6 & 14,21 & 1076 & 89,4 & 14,21 & 904 & 82,9 \\
\hline & 28 & 8 & 9,39 & 14120 & 139,8 & 8,345 & 5935 & 89,7 & 8,14 & 5073 & 82,3 & 8,14 & 5264 & 84,9 \\
\hline & 28 & 10 & 7,54 & 34770 & 247,2 & 6,66 & 26570 & 206,3 & 6,51 & 11410 & 113,9 & 6,51 & 9299 & 99,6 \\
\hline & 30 & 3 & 21,72 & 4437 & 194,9 & 17,34 & 3598 & 269,3 & 17,02 & 2889 & 237,2 & 17,02 & 3989 & 279,3 \\
\hline & 30 & 8 & 12,14 & 114200 & 1343,0 & 10,75 & 17250 & 291,0 & 10,66 & 97400 & 1434,0 & 10,77 & 16090 & 303,5 \\
\hline & 30 & 10 & 7,54 & 30780 & 305,4 & 6,60 & 13560 & 170,7 & 6,55 & 8198 & 120,1 & 6,59 & 8883 & 133,7 \\
\hline & \multicolumn{2}{|c|}{ Average } & 11,10 & 15194 & 164,4 & 9,70 & 5618 & 83,3 & 9,50 & 9294 & 147,6 & 9,60 & 3875 & 75,1 \\
\hline \multirow{16}{*}{$\begin{array}{l}\text { 足 } \\
\text { 岁 } \\
\text { 邑 }\end{array}$} & 15 & 3 & 15,86 & 2248 & 4,4 & 13,58 & 2925 & 6,9 & 13,55 & 2611 & 6,7 & 13,55 & 2388 & 6,7 \\
\hline & 15 & 5 & 7,98 & 877 & 2,7 & 7,35 & 785 & 3,5 & 7,35 & 898 & 3,7 & 7,35 & 762 & 3,4 \\
\hline & 15 & 8 & 6,23 & 726 & 1,1 & 6,14 & 678 & 1,4 & 6,11 & 804 & 1,6 & 6,11 & 698 & 1,5 \\
\hline & 20 & 3 & 17,41 & 4007 & 21,0 & 14,06 & 4384 & 31,1 & 14,06 & 4642 & 31,9 & 14,06 & 4712 & 32,7 \\
\hline & 20 & 8 & 7,40 & 5217 & 15,8 & 7,12 & 4646 & 15,9 & 7,11 & 5109 & 17,8 & 7,11 & 4380 & 15,7 \\
\hline & 20 & 10 & 3,98 & 3505 & 7,8 & 3,91 & 2882 & 8,5 & 3,89 & 3016 & 8,8 & 3,89 & 3319 & 9,5 \\
\hline & 25 & 3 & 16,28 & 4434 & 63,9 & 14,65 & 5324 & 98,9 & 14,65 & 4923 & 91,3 & 14,65 & 6696 & 102,0 \\
\hline & 25 & 8 & 8,07 & 7836 & 64,9 & 7,39 & 4695 & 51,8 & 7,39 & 5066 & 54,5 & 7,39 & 5400 & 57,9 \\
\hline & 25 & 10 & 9,99 & 35520 & 158,8 & 9,96 & 34470 & 151,7 & 9,96 & 49260 & 222,5 & 9,96 & 37030 & 169,3 \\
\hline & 28 & 3 & 17,11 & 5934 & 210,7 & 14,05 & 4436 & 229,0 & 14,05 & 5004 & 290,9 & 14,05 & 3642 & 206,0 \\
\hline & 28 & 8 & 9,14 & 43450 & 580,5 & 8,61 & 54300 & 1787,0 & 8,61 & 35590 & 559,7 & 8,61 & 32250 & 503,8 \\
\hline & 28 & 10 & 8,61 & 140900 & 1323,0 & 8,20 & 94410 & 1013,0 & 8,20 & 105800 & 1150,0 & 8,20 & 125300 & 1328,0 \\
\hline & 30 & 3 & 22,20 & 9661 & 517,0 & 18,45 & 7648 & 623,9 & 18,45 & 7766 & 637,6 & 18,45 & 6555 & 539,6 \\
\hline & 30 & 8 & 12,74 & 187400 & $4854,0^{(3)}$ & 12,26 & 177000 & $4807,0^{(3)}$ & 12,17 & 176000 & $4400,0^{(2)}$ & 12,19 & 184600 & $4494,0^{(2)}$ \\
\hline & 30 & 10 & 9,30 & 253000 & $3586,0^{(1)}$ & 9,06 & 191400 & $2931,0^{(1)}$ & 9,04 & 208800 & $3333,0^{(1)}$ & 9,04 & 194700 & $2991,0^{(1)}$ \\
\hline & \multicolumn{2}{|c|}{ Aver } & 11,50 & 46981 & 760,8 & 10,30 & 39332 & 784,0 & 10,30 & 41019 & 720,7 & 10,30 & 40829 & 697,4 \\
\hline \multicolumn{3}{|c|}{ OTAL } & 11,70 & 22470 & 266,2 & 10,40 & 16108 & 231,6 & 10,30 & 16536 & 230,7 & 10,30 & 16197 & 217,6 \\
\hline
\end{tabular}

